\title{
Exploring Nonlinearities in Financial Systemic Risk
}

\author{
Marcin Wolski ${ }^{1,2}$ \\ ${ }^{1}$ Center for Nonlinear Dynamics in Economics and Finance (CeNDEF), \\ University of Amsterdam, Valckenierstraat 65-67, 1018 XE Amsterdam, NL \\ ${ }^{2}$ Bielefeld Graduate School of Economics and Management (BiGSEM), \\ Bielefeld University, Universitätsstraße 25, D-33615 Bielefeld, DE
}

November 2013

\begin{abstract}
We propose a new methodology of assessing the effects of individual institution's risk on the others and on the system as a whole. We build upon the Conditional Valueat-Risk approach, however, we introduce the explicit Granger causal linkages and we account for possible nonlinearities in the financial time series. Conditional Value-at-RiskNonlinear Granger Causality, or NCoVaR as we call it, has regular asymptotic properties which makes it particularly appealing for practical applications. We test our approach empirically and assess the contribution of the euro area financial companies to the overall systemic risk. We find that only a few financial institutions pose a serious ex ante threat to the systemic risk, whereas, given that the system is already in trouble, there are more institutions which hamper its recovery. Moreover, we discover non-negligible nonlinear structures in the systemic risk profile of the euro zone.
\end{abstract}

JEL Codes: C1, G01, G20, G32

Keywords: Systemic Risk, Financial Risk, Nonparametric Methods

\section{Introduction}

The 2007-2009 crisis shed new light on the complexity within the financial sector. The linkages and risk exposures between various institutions proved to be of great significance in transmitting distress across the whole financial system. Additionally, during systemic events the

\footnotetext{
The author would like to thank Cees Diks and participants of the seminars at the University of Amsterdam for their useful comments. Numerical simulations, reported in this paper, were run on the Lisa Compute Cluster. The author acknowledges also the financial support from the European Doctorate in Economics Erasmus Mundus. The corresponding e-mail addresses is M.Wolski@uva.nl.
} 
malaise spreads across the financial world rapidly through indirect channels, like price effects or liquidity spirals (Brunnermeier, 2009). In effect, market values of various financial assets tend to move closer together, drifting away from their fundamentals. In particular, one observes high regularities in their tail co-movements (Adrian and Brunnermeier, 2011).

Because of its strong adverse effects on the real economy, great attention has been paid to measuring and monitoring systemic risk, i.e. risk that the entire financial system collapses, and individual risk exposures. The majority of econometric approaches in these fields focus on co-risk measures, where the risk of the financial system is assessed in relation to the risk of individual institutions. The intuition behind these models lies in negative externalities which one institution imposes on the others and on the system as a whole. As argued by Adrian and Brunnermeier (2011), these externalities are a consequence of excessive risk taking and leverage. Given, for instance, that one institution is facing a liquidity shock, it liquidates its assets at fire-sale prices as given, affecting borrowing constraints of others and actually causing the fire-sale prices. A wonderful summary of research in this field might be found in Acharya (2009), Acharya et al. (2010) or Adrian and Brunnermeier (2011).

A commonly used econometric approach, in the growing body of literature on this topic, is Conditional Value-at-Risk (CoVaR), attributed to Adrian and Brunnermeier (2011). It is built around the concept of Value-at-Risk (VaR), which determines the maximum loss on returns within the $\gamma$-percentile confidence interval (Kupiec, 2002). CoVaR assesses $\operatorname{VaR}_{\gamma}$ of one institution conditional on a distress in the other. In particular, given that the former represents the system, one may associate $\mathrm{CoVaR}$ with the systemic risk measure.

A clear shortcoming of such an approach lies in its susceptibility to model misspecification. Imagine, that returns come from an unknown probability distribution $F$, with density $f$. Assume now that $f$ is steeper or nonlinear around its $\operatorname{VaR}_{\gamma}$. Clearly, standard parametric approaches oversee this irregularity so that even a small variation in $\mathrm{VaR}_{\gamma}$ might affect co-risk results. In this paper we develop a methodology which corrects for this shortcoming, contributing to the discussion on nonlinear economic dynamics in systemic risk.

The existence of nonlinearities in the field has been already recognized. Huang et al. (2010) suggest that a bank's contribution to the systemic risk is roughly linear in its default probability and highly nonlinear with respect to institution size and asset correlation. This is supported by empirical observations of the financial markets in He and Krishnamurthy (2012). In fact, He and Krishnamurthy (2012) build a theoretical model which matches nonlinear dynamics across different economic variables, including systemic risk. XiaoHua and Shiying (2012) investigate the topic from the neural network perspective and design the early warning mechanism accordingly. This paper aims to propose a formal approach to assess the relevance of nonlinearities in driving systemic events.

We build our approach around the intuition of CoVaR. In particular, we focus on the Granger causal effect that a distress in one institution changes to a distress in the other or in the whole system, where a distress is defined by $\operatorname{VaR}_{\gamma}$. 
There are two main novelties in our methodology. The first one is the notion of causality. The basic CoVaR does not distinguish between direct causal and common factor effects. Adrian and Brunnermeier (2011) treat it as a virtue rather than a problem, arguing that common factor effects are of more importance when dealing with systemic risk, which shall be particularly true for the herding behavior (Brunnermeier et al., 2009). One may, however, want to study the causal relations explicitly. Imagine for instance a group of the biggest financial institutions. Since they do not only trade with each other but also serve as clearing houses or liquidity backstops for smaller parties, they are central to the financial system. Now, imagine that one of them is in trouble. It affects all the banks which are exposed to its risk, but since it is relatively large its distress might alone translate into problems in the entire financial system. The causal kind of reasoning seems therefore particularly appealing for policy makers and central bankers who in fact might want to focus on preventing this individual causal relation.

Another justification for considering causality in individual and systemic risk lies in its possible applications to networks and contagion analysis (see for instance Chinazzi and Fagiolo (2013)). Looking at any pair of institutions, the possible risk effects of one on another do not have to be bilaterally equal (as they are assumed to be in a non-causal setting). For instance, a lender has different kind of risk exposure to a creditor than the other way around. Causality captures that phenomenon explicitly allowing for a more detailed analysis on network spillovers, cascades and shock propagation.

In our study we employ the causality of Granger type (Granger, 1969), as it is intuitive and does not bring many model restrictions. It has been also successfully applied as a network mapping tool in financial analysis (Gao and Ren, 2013).

The second novelty lies in the definition of financial distress. In our study we assume that an institution is in trouble when it is around its $\mathrm{VaR}_{\gamma}$. Practically speaking, our definition captures the majority of events which fall below $\mathrm{VaR}_{\gamma}$ together with some of the events above it. The reason why we allow for some variation around $\mathrm{VaR}_{\gamma}$ lies in its possible nonlinear structure, whose role we want to study explicitly. We recognize that our definition might not capture some of the extreme values from the left tail of the distribution, being potentially susceptible to black swans (Taleb, 2010). Our analysis shows, however, that the optimal region around $\mathrm{VaR}_{\gamma}$ is very slowly decreasing with the sample size, somehow hampering the risk of neglecting the extreme events.

In our analysis we consider two scenarios of potential Granger causality. In the first setting we investigate the role of individual institutions in blocking the recovery of the system which is already under distress. In the second scenario we measure the contribution of individual institutions to the systemic troubles. The second setting is more similar to the standard understanding of systemic risk (Acharya, 2009) and might be useful in ex ante applications. The first scenario might be perceived either as a kind of a robustness check or a policy relevant tool for ex post actions. Indeed, if the system is already in trouble one may want to determine which of its parts are hampering its recovery. In fact, we could think of these two scenarios from 
a perspective of a doctor who prescribes precautionary drugs or is trying to heal an already sick patient.

The paper is organized as follows. In Section 2 we explain the methodology of Conditional Value-at-Risk-Nonlinear Granger Causality (or NCoVaR for simplicity). We evaluate asymptotic properties of the test statistic and we confirm them numerically. In Section 3 we apply our approach to the euro zone financial sector and evaluate which institutions got the most significant impact on the systemic risk in years 2000-2012. Section 4 concludes.

\section{Methodology of NCoVaR}

Let us first bring some intuition behind the Conditional Value-at-Risk and Granger causality separately and then combine them to build CoVaR-NGraCo (Conditional Value-at-RiskNonlinear Granger Causality) or NCoVaR for simplicity. In the standard setting we consider two institutions, $i$ and $j$, whose returns on assets are given by $X^{i}$ and $X^{j}$, respectively. Talking about systemic risk, we set $j$ to be some aggregate variable so that we investigate the relationship between institution $i$ and the system as a whole. Following the original CoVaR literature, let us define $\mathrm{VaR}_{\gamma}$ as the left $\gamma$-quantile of the returns of a given institution. (In practice $\gamma$ is chosen from $\{0.01,0.05,0.1\}$.) For institution $i$ we have therefore

$$
P\left(X^{i} \leq \operatorname{VaR}{ }_{\gamma}^{i}\right)=\gamma
$$

or equivalently

$$
\operatorname{VaR} R_{\gamma}^{i}=\inf \left\{x^{i}: F_{X^{i}}\left(x^{i}\right) \geq \gamma\right\}
$$

where $F_{X^{i}}$ is the cumulative distribution function of $X^{i}$. (For institution $j$, the notation is analogous throughout the paper.) The intuition behind $\mathrm{CoVaR}$ is to evaluate $\mathrm{VaR}_{\gamma}$ of institution $j$ conditional on some event associated with institution $i$. In particular, Adrian and Brunnermeier (2011) consider two conditioning events, i.e. institution $i$ is at its $\operatorname{VaR}_{\gamma}^{i}$ or at its median $\left(\operatorname{VaR}_{\gamma=0.5}^{i}=\right.$ Median $\left.^{i}\right)$. By comparing the difference between the two, it is possible to estimate the risk contribution of institution $i$ onto $j$, denoted by $\Delta$ CoVaR.

In our study we follow similar reasoning as in Adrian and Brunnermeier (2011), however, we add a (discrete) time dimension. For any period $t$, let us define the future returns by $\mathcal{G} X_{t}^{i}$, and past and/or current returns by $\mathcal{F} X_{t}^{i}$. Following Granger (1969), we say that returns of institution $i$ are Granger causing those of institution $j$ if $\mathcal{F} X_{t}^{i}$ contains additional information on $\mathcal{G} X_{t}^{j}$ which is not already contained in $\mathcal{F} X_{t}^{j}$ alone. We formulate the definition of conditional Granger causality analogously, i.e. we say that returns of institution $i$ are Granger causing those of institution $j$ if, conditional on some past or current events $\mathcal{A}\left(\mathcal{F} X_{t}^{i}\right)$ and $\mathcal{B}\left(\mathcal{F} X_{t}^{j}\right)$, $\mathcal{F} X_{t}^{i}$ contains additional information on $\mathcal{G} X_{t}^{j}$ which is not already contained in $\mathcal{F} X_{t}^{j}$ alone.

Given the intuition behind the CoVaR and conditional Granger causality, we may now turn 
to NCoVaR. Similarly to $\Delta$ CoVaR, we test the difference in Granger causal risk effects from institution $i$ on $j$, between two conditioning events, i.e. when institution $i$ is or was in trouble (or around its $\operatorname{VaR}_{\gamma}^{i}$ ) and when it is and was around the median of its returns. An advantage of allowing institutions to be around (and not exactly at) their $\mathrm{VaR}_{\gamma}$ or median levels is that we could thereof account for possible nonlinearities in corresponding distributions - something the original methodology could not capture. In particular, we consider $\mu$-radius ball $(\mu>0)$ centered at $\mathrm{VaR}_{\gamma}$ or median. (The following reasoning holds for $\mathcal{G}$ and $\mathcal{F}$ being multivariate, provided that $\mathrm{VaR}_{\gamma}$ and medians are taken over the marginals.) We allow also for conditioning on the past and/or current realizations of $X_{t}^{j}$. To put it formally, let us formulate the following definition of NCoVaR.

Definition 1. Given any stationary bivariate process $\left\{\left(X_{t}^{i}, X_{t}^{j}\right)\right\}$, we say that $\left\{X_{t}^{i}\right\}$ is a nonlinear CoVaR Granger cause of $\left\{X_{t}^{j}\right\}$ if

$$
P\left(\mathcal{G} X_{t}^{j} \stackrel{\mu}{\approx} \operatorname{VaR}_{\gamma}^{j} \mid \mathcal{F} X_{t}^{i} \stackrel{\mu}{\approx} \operatorname{VaR}_{\gamma}^{i}, \mathcal{B}\left(\mathcal{F} X_{t}^{j}\right)\right) \neq P\left(\mathcal{G} X_{t}^{j} \stackrel{\mu}{\approx} \operatorname{VaR}_{\gamma}^{j} \mid \mathcal{F} X_{t}^{i} \stackrel{\mu}{\approx} \operatorname{Median}^{i}, \mathcal{B}\left(\mathcal{F} X_{t}^{j}\right)\right),
$$

where $\approx^{\mu}$ represents a $\mu$-radius ball, $\mathcal{G}$ denotes future and $\mathcal{F}$ denotes past and/or current states of the corresponding variables and $\mathcal{B}($.$) reflects some event over the argument.$

In this study, we consider two possible scenarios. In the first one, we assume that institution $j$ is already in distress, so that potential Granger causal risk effects from institution $i$ do not only induce even higher losses on $j$ but also can clog its recovery. The second scenario is more similar to the traditional risk analysis, where future troubles in institution $j$ come directly from the past problems of institution $j$. One may thereof reformulate Def. 1 in form of possible two scenarios, which we investigate throughout this paper.

Scenario 1. Given any stationary bivariate process $\left\{\left(X_{t}^{i}, X_{t}^{j}\right)\right\}$, we say that $\left\{X_{t}^{i}\right\}$ is a nonlinear CoVaR Granger cause of $\left\{X_{t}^{j}\right\}$ in tail if

$$
\begin{gathered}
P\left(\mathcal{G} X_{t}^{j} \stackrel{\mu}{\approx} \operatorname{VaR}_{\gamma}^{j} \mid \mathcal{F} X_{t}^{i} \stackrel{\mu}{\approx} \operatorname{VaR}_{\gamma}^{i}, \mathcal{F} X_{t}^{j} \stackrel{\mu}{\approx} \operatorname{VaR}_{\gamma}^{j}\right) \neq \\
P\left(\mathcal{G} X_{t}^{j} \stackrel{\mu}{\approx} \operatorname{VaR}_{\gamma}^{j} \mid \mathcal{F} X_{t}^{i} \stackrel{\mu}{\approx} \operatorname{Median}^{i}, \mathcal{F} X_{t}^{j} \stackrel{\mu}{\approx} \operatorname{VaR}_{\gamma}^{j}\right),
\end{gathered}
$$

where $\approx^{\mu}$ represents a $\mu$-radius ball, $\mathcal{G}$ denotes future and $\mathcal{F}$ denotes past and/or current states of the corresponding variables.

Scenario 2. Given any stationary bivariate process $\left\{\left(X_{t}^{i}, X_{t}^{j}\right)\right\}$, we say that $\left\{X_{t}^{i}\right\}$ is a nonlinear CoVaR Granger cause of $\left\{X_{t}^{j}\right\}$ in median if

$$
\begin{array}{r}
P\left(\mathcal{G} X_{t}^{j} \stackrel{\mu}{\approx} \operatorname{VaR}_{\gamma}^{j} \mid \mathcal{F} X_{t}^{i} \stackrel{\mu}{\approx} \operatorname{VaR}_{\gamma}^{i}, \mathcal{F} X_{t}^{j} \stackrel{\mu}{\approx} \operatorname{Median}^{j}\right) \neq \\
P\left(\mathcal{G} X_{t}^{j} \stackrel{\mu}{\approx} \operatorname{VaR}_{\gamma}^{j} \mid \mathcal{F} X_{t}^{i} \stackrel{\mu}{\approx} \operatorname{Median}^{i}, \mathcal{F} X_{t}^{j} \stackrel{\mu}{\approx} M_{\text {edian }}^{j}\right),
\end{array}
$$

where $\approx^{\mu}$ represents a $\mu$-radius ball, $\mathcal{G}$ denotes future and $\mathcal{F}$ denotes past and/or current states 
of the corresponding variables.

In practice it is impossible to condition of the infinite sets of future or past realizations of variables of interest. Therefore, we reformulate $\mathcal{G}$ and $\mathcal{F}$ as finite sets of future periods or lags, respectively. We limit ourselves to the canonical setting where $\mathcal{G} X_{t}^{j}=X_{t+1}^{j}$, as it is most commonly used in practical Granger causality testing, however, our reasoning holds for any $\mathcal{G} X_{t}^{j}=X_{t+k}^{j}, 1 \leq k<\infty$. Similarly, we replace $\mathcal{F} X_{t}^{i}$ and $\mathcal{F} X_{t}^{j}$ by $X_{t, l_{i}}^{i}=\left\{X_{t-l_{i}+1}^{i}, \ldots, X_{t}^{i}\right\}$ and $X_{t, l_{j}}^{j}=\left\{X_{t-l_{j}+1}^{j}, \ldots, X_{t}^{j}\right\}$, where $l_{i} \geq 1, l_{j} \geq 1$ denote the number of lags of a corresponding variable.

In Granger causality testing, the goal is to find evidence against the null hypothesis of no causality, which according to Def. 1 is represented by equivalence in conditional probability. For a strictly stationary process $\left\{\left(X_{t}^{i}, X_{t}^{j}\right)\right\}$, the null is the statement about the invariant distribution in conditional $\operatorname{VaR}_{\gamma}$ levels of the $\left(l_{i}+l_{j}+1\right)$-dimensional vector $W_{t}=\left(Z_{t}, X_{t, l_{i}}^{i}, X_{t, l_{j}}^{j}\right)$, where we substitute $Z_{t}=X_{t+1}^{j}$. (For clarity purposes and to bring forward the fact that we consider the invariant distribution of $W_{t}$, we drop the time index, so that $W=\left(Z, X^{i}, X^{j}\right)$.) It is helpful to restate the problem in terms of ratios of joint densities evaluated at given quantiles as under the null the density of $Z$ evaluated around its $\mathrm{VaR}_{\gamma}$ level and conditional on specific events in $X^{i}$ and $X^{j}$ is equal the same density conditional on the different set of events in $X^{i}$ and $X^{j}$. Therefore, the joint probability density function, together with its marginals must satisfy

$$
\frac{f_{Z, X^{i}, X^{j}}\left(z_{\gamma}, x_{\gamma}^{i}, x_{*}^{j}\right)}{f_{X^{i}, X^{j}}\left(x_{\gamma}^{i}, x_{*}^{j}\right)}=\frac{f_{Z, X^{i}, X^{j}}\left(z_{\gamma}, x_{m}^{i}, x_{*}^{j}\right)}{f_{X^{i}, X^{j}}\left(x_{m}^{i}, x_{*}^{j}\right)},
$$

where $z_{\gamma}=\operatorname{VaR}_{\gamma}^{Z}, x_{\gamma}^{i}=\operatorname{VaR}_{\gamma}^{i}, x_{m}^{i}=$ Median ${ }^{i}$ and $*$ distinguishes between Scenario 1 and 2 as $x_{\gamma}^{j}=\operatorname{VaR}_{\gamma}^{j}$ or $x_{m}^{j}=$ Median ${ }^{j}$, respectively. Since Eq. (3) holds for any quantile of the vector $\left(Z, X^{i}, X^{j}\right)$ in the support of $Z, X^{i}, X^{j}$, Eq. (3) might be equivalently rewritten as

$$
\frac{f_{Z, X^{i}, X^{j}}\left(z_{\gamma}, x_{\gamma}^{i}, x_{*}^{j}\right)}{f_{X^{i}, X^{j}}\left(x_{m}^{i}, x_{*}^{j}\right)}=\frac{f_{X^{i}, X^{j}}\left(x_{\gamma}^{i}, x_{*}^{j}\right)}{f_{X^{i}, X^{j}}\left(x_{m}^{i}, x_{*}^{j}\right)} \frac{f_{Z, X^{i}, X^{j}}\left(z_{\gamma}, x_{m}^{i}, x_{*}^{j}\right)}{f_{X^{i}, X^{j}}\left(x_{m}^{i}, x_{*}^{j}\right)} .
$$

Natural methodology to assess Eq. (4) comes from the Baeck and Brock (1992) test for conditional independence, or its Granger causal version from Hiemstra and Jones (1994). However, as showed by Diks and Panchenko (2005) and Diks and Panchenko (2006), these tests can severely over-reject in Granger causal setting, because its dependence on the conditional variance. Diks and Panchenko (2006) propose to add a positive weight function $g\left(z, x^{i}, x^{j}\right)$ and, given that the null should hold in the support of the joint densities, it might be equivalently 
written as

$$
\begin{aligned}
\tau_{g} & \equiv\left(\frac{f_{Z, X^{i}, X^{j}}\left(z_{\gamma}, x_{\gamma}^{i}, x_{*}^{j}\right)}{f_{X^{i}, X^{j}}\left(x_{m}^{i}, x_{*}^{j}\right)}\right. \\
& \left.-\frac{f_{X^{i}, X^{j}}\left(x_{\gamma}^{i}, x_{*}^{j}\right)}{f_{X^{i}, X^{j}}\left(x i_{m}, x_{*}^{j}\right)} \frac{f_{Z, X^{i}, X^{j}}\left(z_{\gamma}, x_{m}^{i}, x_{*}^{j}\right)}{f_{X^{i}, X^{j}}\left(x_{m}^{i}, x_{*}^{j}\right)}\right) g\left(z, x^{i}, x^{j}\right)=0 .
\end{aligned}
$$

Diks and Panchenko (2006) discuss several possibilities of choosing $g\left(z, x^{i}, x^{j}\right)$. In this study we focus on $g\left(z, x^{i}, x^{j}\right)=f_{X^{i}, X^{j}}\left(x_{m}^{i}, x_{*}^{j}\right)^{2}$, as the estimator of $\tau_{g}$ has a corresponding U-statistic representation, bringing the desired asymptotic normality properties for weakly dependent data. Substituting into Eq. (5), one finds that

$$
\tau=f_{Z, X^{i}, X^{j}}\left(z_{\gamma}, x_{\gamma}^{i}, x_{*}^{j}\right) f_{X^{i}, X^{j}}\left(x_{m}^{i}, x_{*}^{j}\right)-f_{X^{i}, X^{j}}\left(x_{\gamma}^{i}, x_{*}^{j}\right) f_{Z, X^{i}, X^{j}}\left(z_{\gamma}, x_{m}^{i}, x_{*}^{j}\right) .
$$

To evaluate the data driven representation of $\tau$, we rely on kernel methods. In particular, we consider the local density estimator

$$
\hat{f}_{W}(w)=\frac{\varepsilon^{-d_{W}}}{n} \sum_{k=1}^{n} K\left(\frac{w-w_{k}}{\varepsilon}\right),
$$

where $n$ is the sample size, $\varepsilon$ is the bandwidth parameter (similar to $\mu$ from the Def. 1 ), $d$ reflects the dimensionality of a given vector $W$ and $K($.$) is a bounded Borel function \mathbb{R}^{d_{W}} \rightarrow \mathbb{R}$ satisfying

$$
\int|K(t)| d t<\infty, \quad \int K(t) d t=1 \quad \text { and } \quad|t K(t)| \rightarrow 0 \quad \text { as } \quad|t| \rightarrow \infty .
$$

In practice, $K($.$) is often chosen to be a probability density function (Wand and Jones, 1995).$ In order to guarantee the consistency of the pointwise density estimators, we assume that the bandwidth parameter $\varepsilon$ comes from the sequence $\varepsilon_{n}$, which is slowly decreasing with the sample size, i.e.

$$
\varepsilon_{n} \rightarrow 0 \quad \text { and } \quad n \varepsilon_{n} \rightarrow \infty \quad \text { as } n \rightarrow \infty
$$

Parzen (1962) shows that under conditions (8) and (9) and provided that $f$ is continuous at $w$, the estimate of density $f$ at a given point $w$ is consistent. 
Given a given bandwidth $\varepsilon$, a natural estimator for $\tau$ is found to be

$$
\begin{aligned}
T_{n}(\varepsilon) & =C \sum_{k=1}^{n} \sum_{p=1}^{n}\left[K\left(\frac{\left(z_{\gamma}, x_{\gamma}^{i}, x_{*}^{j}\right)^{T}-\left(z_{k}, x_{k}^{i}, x_{k}^{j}\right)^{T}}{\varepsilon}\right) K\left(\frac{\left(x_{m}^{i}, x_{*}^{j}\right)^{T}-\left(x_{p}^{i}, x_{p}^{j}\right)^{T}}{\varepsilon}\right)\right. \\
& \left.-K\left(\frac{\left(x_{\gamma}^{i}, x_{*}^{j}\right)^{T}-\left(x_{k}^{i}, x_{k}^{j}\right)^{T}}{\varepsilon}\right) K\left(\frac{\left(z_{\gamma}, x_{m}^{i}, x_{*}^{j}\right)^{T}-\left(z_{p}, x_{p}^{i}, x_{p}^{j}\right)^{T}}{\varepsilon}\right)\right],
\end{aligned}
$$

with

$$
C=\frac{\varepsilon^{-d_{Z}-2 d_{X^{i}-2 d_{X^{j}}}}}{n^{2}} .
$$

The asymptotic distribution of the test statistic might be derived from the behavior of the properties of the second order U-statistic, as described in Serfling (1980) and van der Vaart (1998).

Theorem 1. Under conditions (8) and (9), for a given $\mathrm{VaR}_{\gamma}$ levels and given bandwidth parameter sequence $\varepsilon_{n}$, test statistic $T_{n}\left(\varepsilon_{n}\right)$ satisfies:

$$
\sqrt{n} \frac{\left(T_{n}\left(\varepsilon_{n}\right)-\tau\right)}{S_{n}} \stackrel{d}{\longrightarrow} \mathcal{N}(0,1)
$$

where $S_{n}$ is the autocorrelation consistent estimator of the asymptotic standard deviation of $\sqrt{n}\left(T_{n}\left(\varepsilon_{n}\right)-\tau\right)$.

The proof of Theorem 1 might be found in A. As argued by Diks and Panchenko (2006), although the test statistic is not positive definite, the one-sided test, i.e. rejecting on larger values, turns out to yield better performance.

In this study we choose $\gamma$ to be 0.05 as it is most commonly applied VaR significance level. We calculate $\mathrm{VaR}_{\gamma}$ from the empirical quantile function (Jones, 1992). We closely follow the literature on nonparametric Granger causality testing (Hiemstra and Jones, 1994; Diks and Panchenko, 2006) and take the square kernel function. ${ }^{1}$ The square kernel form of the estimator in Eq. (7), can be rewritten as

$$
\hat{f}_{W}^{S Q}(w)=\frac{(2 \varepsilon)^{-d_{W}}}{n-1} \sum_{k=1}^{n} I\left(\left\|w-w_{k}\right\|<\varepsilon\right)
$$

where $I\left(\left\|w-w_{k}\right\|<\varepsilon\right)$ is the indicator function taking values 1 for any $\left\|w-w_{k}\right\|<\varepsilon$ and zero otherwise, and $\|$.$\| is the supremum norm over all the dimensions.$

\footnotetext{
${ }^{1} \mathrm{Up}$ to the scaling factor, the square kernel is parallel to the rectangular (uniform) kernel, with all its moments being similar. The asymptotic properties of the test statistic are, however, robust to any kernel specification, provided that it satisfies conditions (8) and (9).
} 


\subsection{Optimal bandwidth}

Although the asymptotic normality of the test statistic holds for arbitrary decreasing sequence of bandwidths as long as it satisfies condition (9), it influences the power of the test to a great extent (Silverman, 1998). Therefore, in order to improve the performance of the test, we calculate the optimal size of the bandwidth explicitly. Following Wand and Jones (1995) and Silverman (1998), the optimal bandwidth shall minimize the Mean Squared Error (MSE) of $T_{n}\left(\varepsilon_{n}\right)$, which may be decomposed into the sum of variance and squared bias of $T_{n}\left(\varepsilon_{n}\right)$. In our inference it is worthwhile to point out that the optimal bandwidth values of $T_{n}\left(\varepsilon_{n}\right)$ do not violate the consistency properties of any of the density estimators.

Corollary 1. Under conditions (8) and (9), the MSE optimal sequence of bandwidths of $T_{n}\left(\varepsilon_{n}\right)$ guarantee consistency of any of the pointwise density estimators contributing to $T_{n}\left(\varepsilon_{n}\right)$.

The proof of Corollary 1 is given in B. In fact, the optimum rate of convergence of the bandwidth of $T_{n}\left(\varepsilon_{n}\right)$ is slightly faster than these of individual density estimators, but still much slower than $n^{-1}$. This is caused by increased variance of a product of two estimators compared to their individual variances. Therefore, in order to control for this effect, the sequence of optimal bandwidths of $T_{n}\left(\varepsilon_{n}\right)$ should decrease at a slightly faster rate as $n \rightarrow \infty$, but never as fast as $n^{-1}$. In testing for systemic risk this proves to be of large importance as with a bandwidth parameter decreasing just slightly with the sample size we are still able to capture the majority of returns which are left to $\mathrm{VaR}_{\gamma}$.

In evaluating the optimal bandwidth value we rely on the Monte Carlo methods. Correcting for the weak dependency, we apply the autocorrelation consistent estimator for the variance of $T_{n}(\varepsilon)$, as proposed in Newey and West (1987). It might be verified that for a given bandwidth $\varepsilon$, the bias of $T_{n}(\varepsilon)$ might be calculated from the Taylor expansion around any point as

$$
\begin{aligned}
E\left[T_{n}(\varepsilon)\right]-\tau & =\frac{1}{2} \kappa_{2} \varepsilon^{2}\left[f_{Z, X^{i}, X^{j}}\left(z_{r}, x_{r}^{i}, x_{*}^{j}\right) \nabla^{2} f_{X^{i}, X^{j}}\left(x_{s}^{i}, x_{*}^{j}\right)\right. \\
& +f_{X^{i}, X^{j}}\left(x_{s}^{i}, x_{*}^{j}\right) \nabla^{2} f_{Z, X^{i}, X^{j}}\left(z_{r}, x_{r}^{i}, x_{*}^{j}\right) \\
& -f_{X^{i}, X^{j}}\left(x_{r}^{i}, x_{*}^{j}\right) \nabla^{2} f_{Z, X^{i}, X^{j}}\left(z_{r}, x_{s}^{i}, x_{*}^{j}\right) \\
& \left.-f_{Z, X^{i}, X^{j}}\left(z_{r}, x_{s}^{i}, x_{*}^{j}\right) \nabla^{2} f_{X^{i}, X^{j}}\left(x_{r}^{i}, x_{*}^{j}\right)\right]+o\left(\varepsilon^{2}\right),
\end{aligned}
$$

where $\kappa_{2}$ is the second moment of the kernel and $\nabla^{2} f_{W}(w)$ is the trace of the second derivative of density evaluated at point $w$. Up to the error of order $o\left(\varepsilon^{2}\right)$, Eq. (13) has a plug in estimator, which might be easily calculated using kernel methods (Wand and Jones, 1995).

To give an example on the size of the optimal bandwidth value, we perform a bootstrap experiment on the same bivariate process as in the Jeong et al. (2012), i.e.

$$
\begin{aligned}
& x_{t}^{i}=1+\frac{1}{2} x_{t-1}^{i}+r_{1, t} \\
& x_{t}^{j}=\frac{1}{2} x_{t-1}^{j}+c\left(x_{t-1}^{i}\right)^{2}+r_{2, t},
\end{aligned}
$$




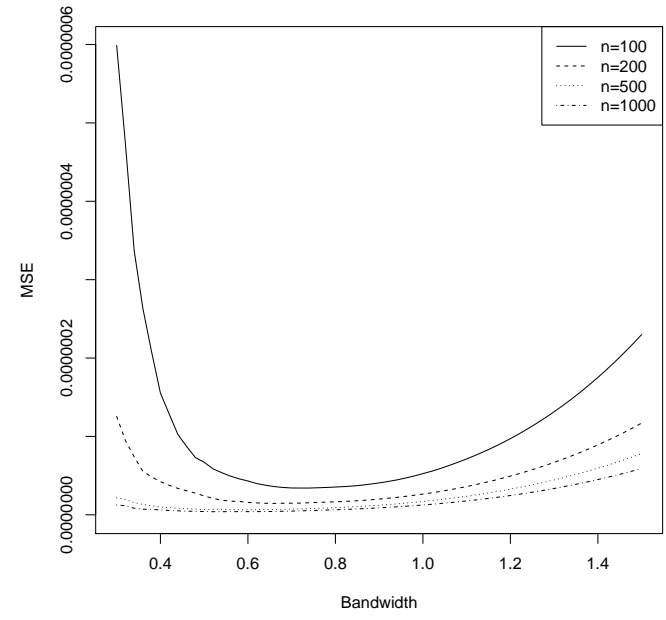

(a) Null hypothesis as in Sc. 1 .

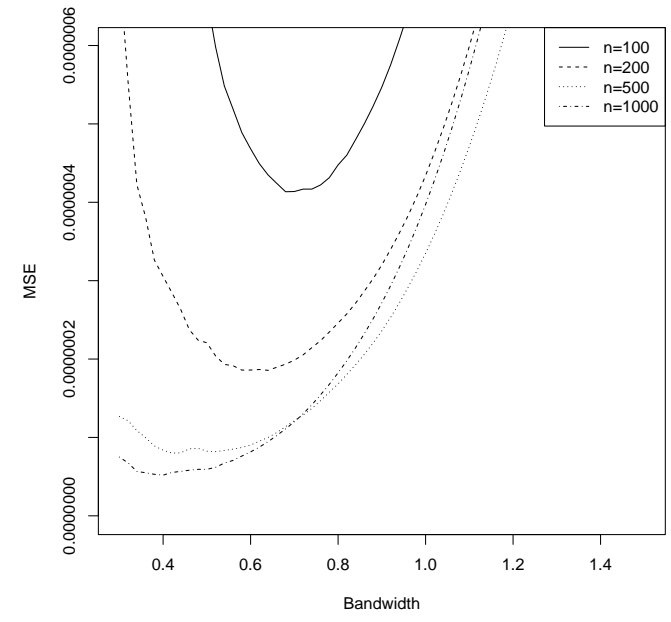

(b) Null hypothesis as in Sc. 2 .

Figure 1: MSE of the test statistic for bandwidth values in the range $[0.3,1.5]$ and for different sample sizes, aggregated over 1000 simulations.

where $r_{1, t}$ and $r_{2, t}$ independent standard normal variables. The biggest advantage of process in Eq. (14) is its tuning parameter on Granger causality, $c$. Clearly, if $c=0$ the model corresponds to the hypothetical scenario of no Granger causality from the first lags of $X_{t}^{i}$ on $X_{t}^{j}$. The larger parameter $c$ becomes, the stronger Granger causal effect, which we may control for explicitly.

We perform 1000 simulations of normalized data of process from Eq. (14) for different sample sizes and evaluate MSE of the test statistic for different bandwidth values from the range $[0.3,1.5]$. For practical reasons, the interval between the bins is taken as 0.01 . We take lags of order 1 for both variables, as suggested by the underlying lag structure in Eq. (14). The results for two scenarios of Granger causality are presented in Fig. 1 and the optimal bandwidths are reported in Table 1 .

It is straightforward to notice the differences of the MSE curves between two settings. Firstly, for the same sample size and $\varepsilon$, Scenario 2 demonstrates larger MSE than in Scenario 1. Secondly, in Scenario 1 the MSE curve becomes flatter, whereas in Scenario 2 the visible U-shape is preserved as the sample size increases. These, in fact, are direct consequences of the curvature of the true distribution around particular quantiles. Scenario 1 is driven by the tail dependence, where the curvature shall be relatively flatter. On the contrary, Scenario 2 represents the relation between the tail and the median, where the distribution shall be more bell-shaped or simply steeper. This, in fact, shows up in the steepness and in the relative size of the MSE curve. As expected, the minimum of the MSE curves is decreasing with the sample size in both scenarios (see Table 1).

Reported optimal bandwidth values represent the radius around the $V a R_{\gamma}$ which is being 
Table 1: Optimal bandwidth values for test statistic evaluated for the process from Eq. (14) for different sample sizes and for two scenarios. The values represent means over 1000 simulations.

\begin{tabular}{lrccc}
\hline \hline & $n=100$ & $n=200$ & $n=500$ & $n=1000$ \\
\hline$\varepsilon^{*}(\mathrm{Sc} .1)$ & 0.74 & 0.66 & 0.6 & 0.52 \\
$\varepsilon^{*}(\mathrm{Sc} .2)$ & 0.68 & 0.64 & 0.48 & 0.44 \\
\hline \hline
\end{tabular}

considered in the NCoVaR. One may readily observe that Scenario 1 has slightly larger bandwidths than Scenario 2. We may view this as a result of scarcity of data in tails compared with that around the median. Extracting information from tails requires, on average, slightly larger windows in comparison to the region near the median (Caers and Maes, 1998).

Bootstrapping optimal bandwidths is a powerful technique which might be applied to any data set without assuming an underlying process structure. We recognize, however, that it might take a lot of computational time. For very large samples we suggest taking bins of 0.02 or 0.05 in order to make it computationally less demanding. Our simulations confirm that the power of the test is preserved in the range $\left[\varepsilon^{*}-0.05, \varepsilon^{*}+0.05\right]$.

\section{$2.2 \quad$ Numerical performance}

We perform two experiments to evaluate the practical side of the test. In both we rely on Monte Carlo methods on the example of the process in Eq. (14). ${ }^{2}$ In the first one, we assess the distribution of the test statistic under the null, evaluated for different sample sizes for 500 runs. In the second experiment, we estimate the power of the test. Given that the null hypothesis is violated $(c>0)$, we estimate rejection rates for different nominal significance levels. We summarize the results from both experiments in the size-size plots and size-adjusted power diagrams. The former plots the actual against nominal cumulative rejection rates under the null, showing the size distortions. The later shows the power of the test corrected for the possible size bias, plotting the observed cumulative rejection rates under the alternative (actual power) against observed rejection rates under the null (actual size). Ideally, the power function shall be 1 for any significance level larger than 0 , however, in practice we would like to observe an increase in the slope at the origin as the sample size grows. Fig. 2 shows the size-size diagrams whereas the size-adjusted power plots are presented in Figs 3-5.

Fig. 2 suggests that the nominal size distortions are larger in Scenario 2 than in Scenario 1. Additionally, the size-size curves are flatter in Scenario 1 whereas they are more wavy in Scenario 2. In fact, this is similar to the pattern observed in the MSE (see Fig. 1) and might be largely attributed to the curvature of the true distribution around particular quantiles.

One may readily observe from Figs 3-5 that the size-adjusted power of the test increases

\footnotetext{
${ }^{2}$ One may expect that the numerical size distortions and power of the NCoVaR test could depend on the exact process specification. Eq. (14) offers a simple testing environment, which has been already applied in the quantile testing literature (Jeong et al., 2012). We leave, therefore, the assessment of the NCoVaR numerical performance on other processes for future investigation.
} 


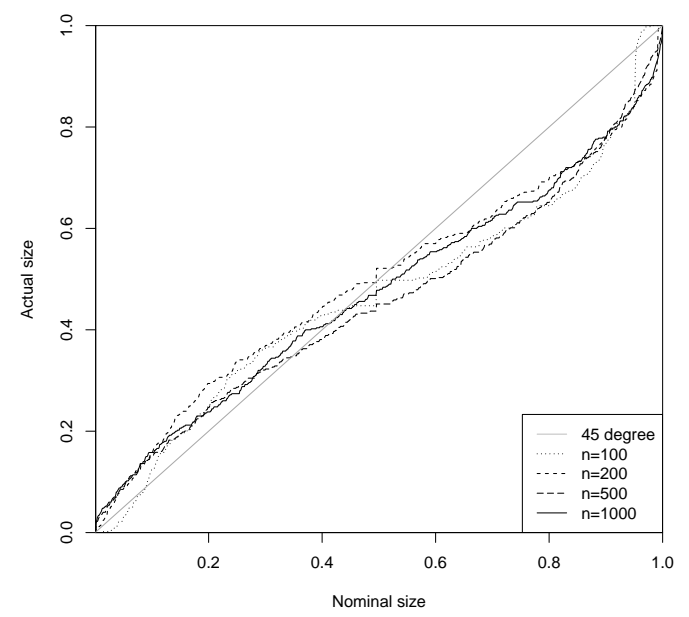

(a) Null hypothesis as in Sc. 1.

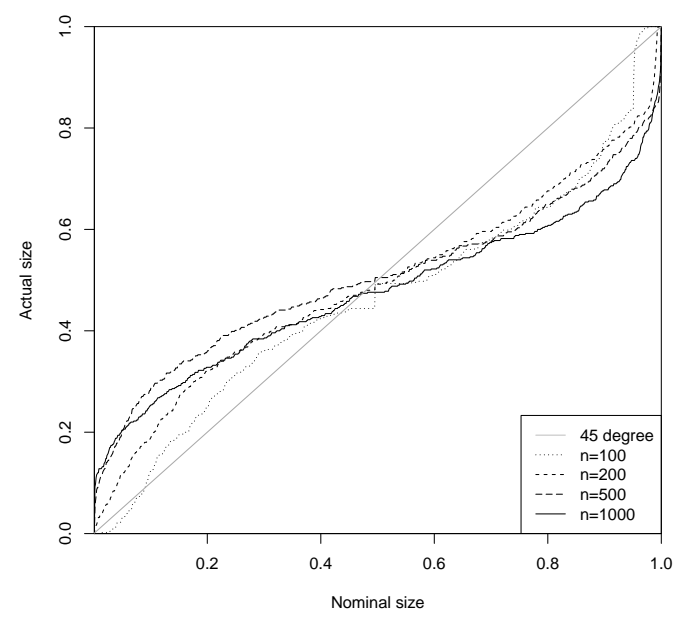

(b) Null hypothesis as in Sc. 2.

Figure 2: Size-size diagram of the NCoVaR test for the process from Eq. (14) for different sample sizes over 500 simulations.

with the sample size and with the strength of Granger causality. Nevertheless, there are two main patterns emerging from the numerical analysis which deserve pointing out.

Firstly, for relatively smaller size the power of the test is higher for Scenario 1 than for Scenario 2. This is again the result of model dynamics, where the underlying relation on variable $j$, i.e. $P\left(X_{t+1}^{j} \approx \operatorname{VaR}_{\gamma}^{j} \mid X_{t}^{j} \approx M e d i a n^{j}\right)$ is more rare to observe on the process from Eq. (14). Practically speaking, as the sample size gets larger this effect is hampered.

Secondly, the size-adjusted test power is almost negligible for very small Granger causality and short time series. Clearly, one shall blame the relative scarcity of observations around quantiles for this discomfort. In order to apply the test to shorter data sets, we propose two solutions to overcome this issue. The first one comprises different kernel specifications. The square kernel takes into account only observations which are $\varepsilon$-close to the quantile, leaving out many possibly informative data points. Replacing kernel by a smoother one, like Gaussian or logistic, should thereof correct for this effect. The second possible solution lies in improving the precision of the density estimators. In the standard kernel estimators (like square kernel estimators applied here) the bias is of order $\varepsilon^{2}$ (Wand and Jones, 1995). Making the bias smaller should decrease the disinformative effect of the observations around a given quantile so that keeping the sample size fixed we get relatively better representation of the true Granger causal relation, which translates into improved test performance.

One may consider Data Sharpening (DS) as being potentially attractive bias reduction method in our setting. Following Hall and Minnotte (2002), the idea behind DS is to slightly perturb the original data set in order to obtain desirable estimator properties (here it is the 


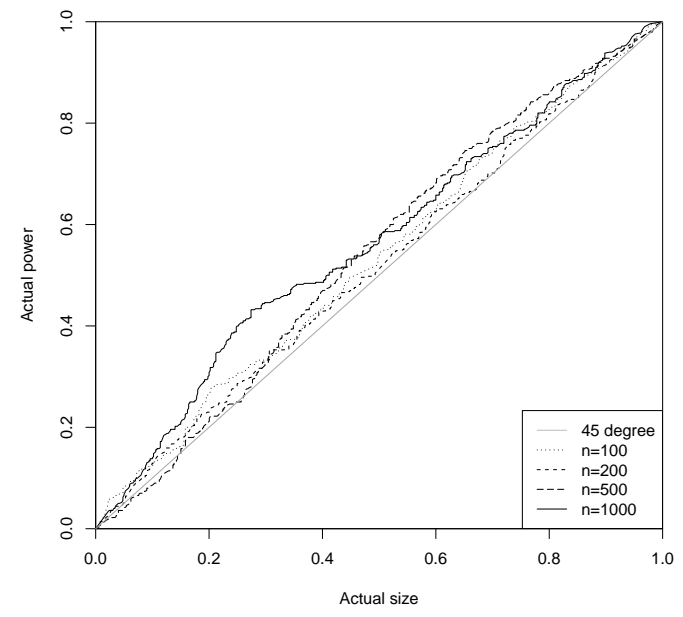

(a) Null hypothesis as in Sc. 1.

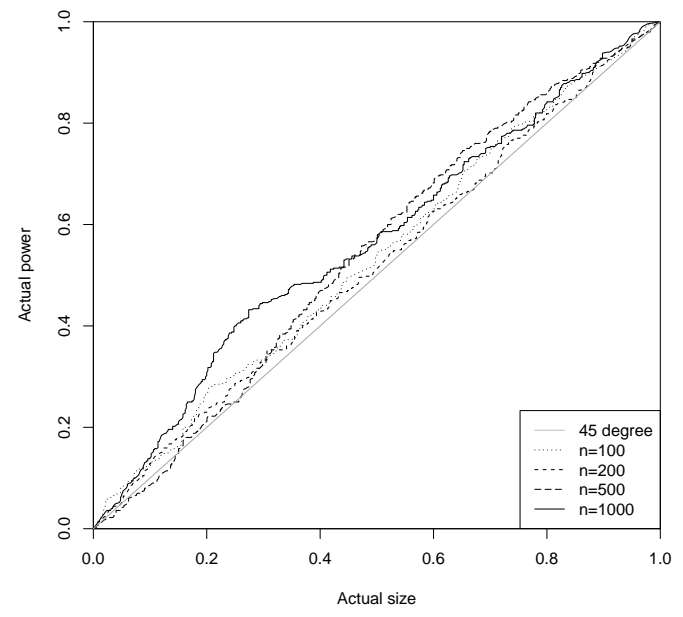

(b) Null hypothesis as in Sc. 2 .

Figure 3: Size-adjusted power for the NCoVaR test for the process from Eq. (14) for $c=0.05$ for different sample sizes over 500 simulations.

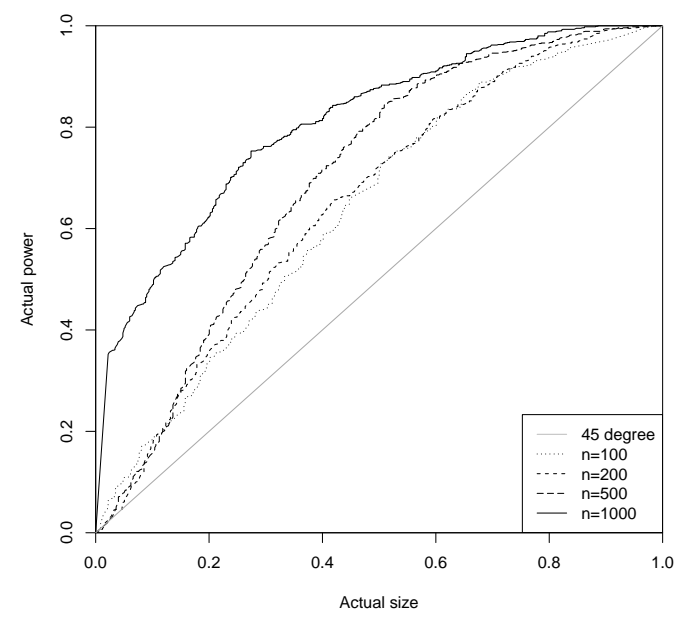

(a) Null hypothesis as in Sc. 1.

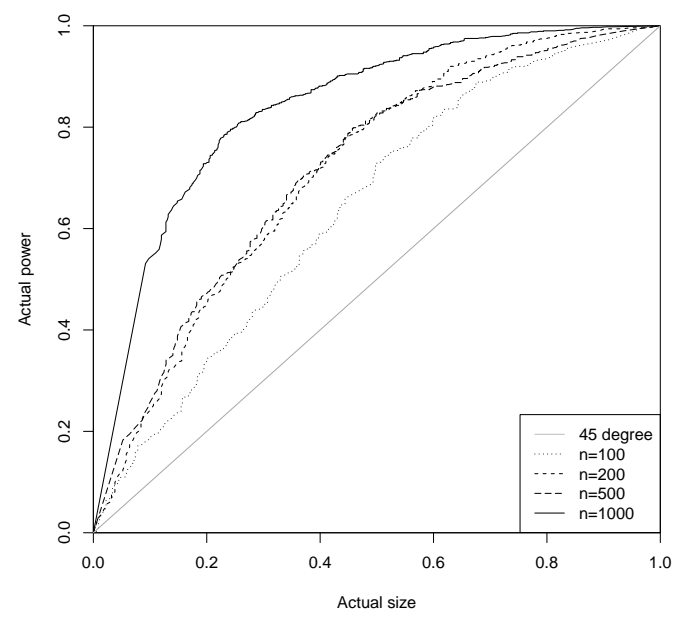

(b) Null hypothesis as in Sc. 2 .

Figure 4: Size-adjusted power for the NCoVaR test for the process from Eq. (14) for $c=0.25$ for different sample sizes over 500 simulations. 


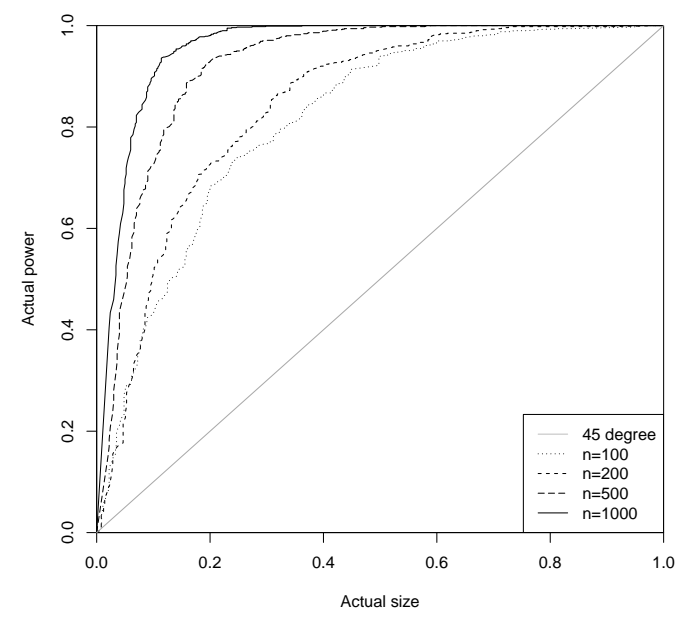

(a) Null hypothesis as in Sc. 1 .

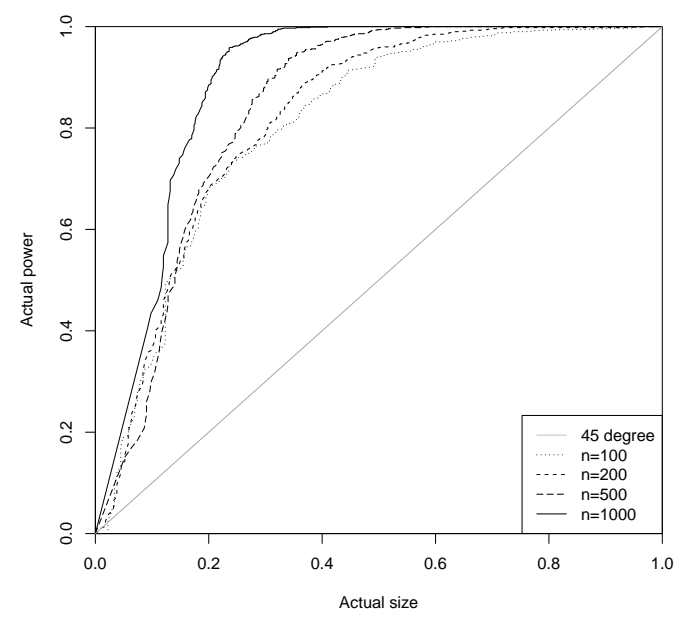

(b) Null hypothesis as in Sc. 2 .

Figure 5: Size-adjusted power for the NCoVaR test for the process from Eq. (14) for $c=0.4$ for different sample sizes over 500 simulations.

reduced bias). Diks and Wolski (2013) show that, besides reducing the estimator bias, DS does not affect other asymptotic properties of the test statistic in a similar Granger causality setting. Therefore, it seems to be a straightforward extension to NCoVaR for shorter samples.

\section{Assessing financial systemic risk}

In our analysis we focus on the $\mathrm{NCoVaR}$ of individual institutions on the overall systemic risk. Thereof, we set that $j$ represents the system variable and $i$ is the individual financial institution.

We approximate the returns on assets by equity returns and take into account financial institutions publicly traded within the euro zone. In order to make the analysis more transparent we focus on the companies which build the Euro STOXX Financial Index in years 2000-2012. Our sample covers thus the Great Recession in Europe (2008-12), the financial crisis (20072009 ) and the sovereign debt crisis (2010-2012). In total we collect daily equity returns for 48 companies (3 financial, 13 insurance, 23 banks and 9 real estate) and one aggregate index. For each variable we have 3390 observations. The list of companies, together with the country of origin and their sector might be found in D. The data have been obtained from the DataStream.

All time series are stationary at the $1 \%$ significance level, according to both Phillips-Perron and Augmented Dickey-Fuller specifications (Phillips and Perron, 1988; Fuller, 1995). We run the pairwise tests against the null of no NCoVaR between each company and system variable. 


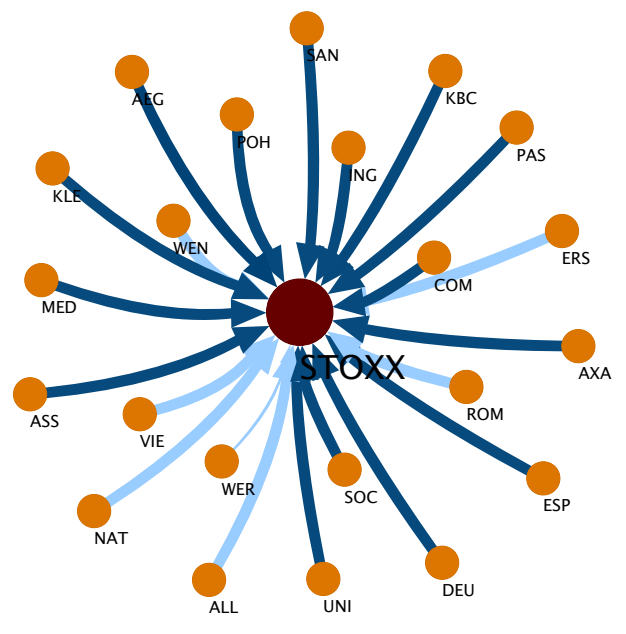

(a) Euro area NCoVaR in Sc. 1.

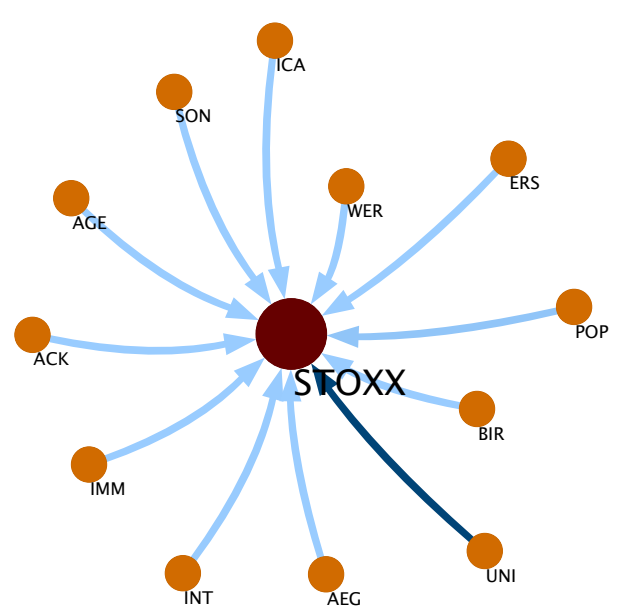

(b) Euro area NCoVaR in Sc. 2.

Figure 6: NCoVaR between euro area individual financial companies and system variable for raw data.

In order to make sure that all the Granger causal relations are nonlinear, we run the same test specification on VAR-filtered residuals also. In each run the number of lags is taken in accordance with the Schwarz-Bayes Information Criterion from the VAR specification and the optimal bandwidth value is approximated by bootstrap. As a robustness check, we also correct for possible causality in second moments, as suggested in Francis et al. (2010), by running NCoVaR test on residuals from Dynamics Conditional Correlation GARCH model (Engle, 2002).

The detailed results can be found in D (Tables D.2, D.3 and D.4), however, for presentational clarity we refer to the star-graphs, which show the NCoVaR between each company and system as a whole. Width of the arrows represents the inverse of the statistical significance level of NCoVaR (the stronger the NCoVaR effect, the wider (and darker) the arrow). Fig. 6 shows the results for the raw time series, Fig. 7 depicts the VAR-filtered returns and Fig. 8 refers to the GARCH residuals.

Considering that at least one NCoVaR relation denotes a systemically important institution, our analysis suggests that out of 48 companies 33 of them might be so described. The group consists of 3 financial services companies, 6 insurance firms, 19 banks and 5 real estate companies. In fact, all of the financial services companies in our sample prove to be systemically important.

There are two main patterns emerging from our analysis. Firstly, there are fewer systemically risky institutions in Scenario 2. Secondly, NCoVaR in Scenario 1 is on average stronger than in Scenario 2. These findings hold for the original and VAR- and GARCH-filtered data. 


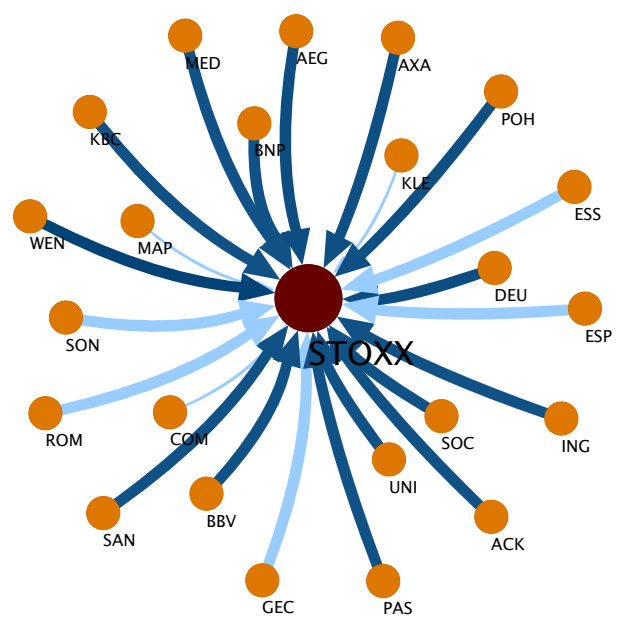

(a) Euro area NCoVaR in Sc. 1.

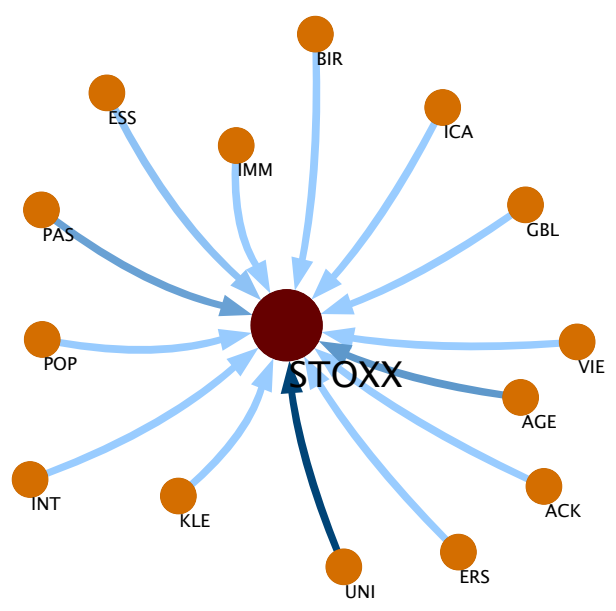

(b) Euro area NCoVaR in Sc. 2 .

Figure 7: NCoVaR between euro area individual financial companies and system variable for VAR-filtered data.

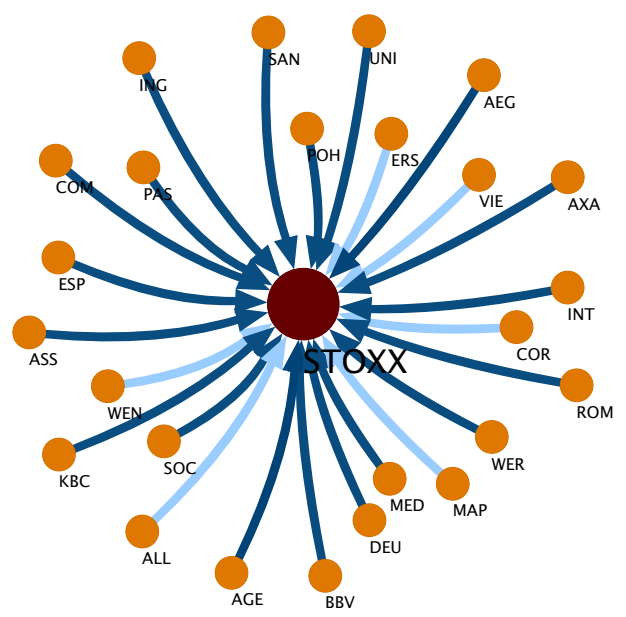

(a) Euro area NCoVaR in Sc. 1.

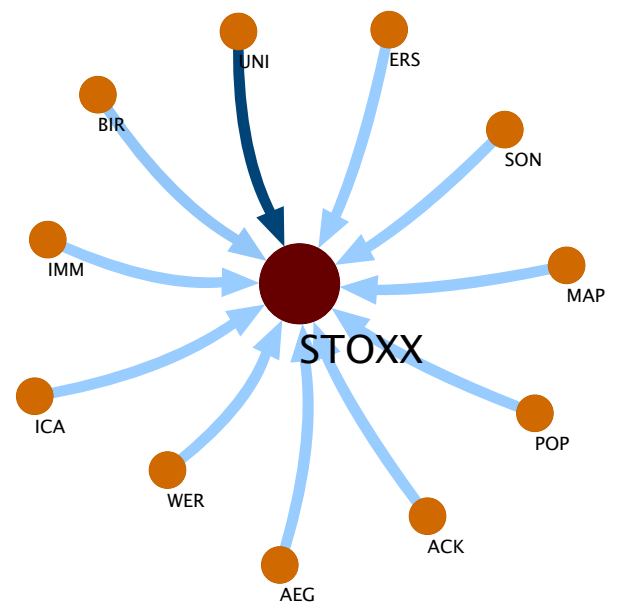

(b) Euro area NCoVaR in Sc. 2.

Figure 8: NCoVaR between euro area individual financial companies and system variable for GARCH-filtered data. 
Interestingly, our study suggests that only a few financial institutions pose a serious ex ante threat to the systemic risk in the euro area, whereas, given that the system is already in trouble, there are more institutions which hamper its recovery. This result confirms a common view in the literature on macro-prudential supervision (Acharya, 2009) that the relative preventive costs are smaller than those after the crisis has already erupted.

The analysis confirms the nonlinear structure of the institutional contribution to the systemic risk. Filtering out the linear relations and second moment influence does not remove the co-risk relations among individual companies and system as a whole. Interestingly, after filtering we observe some new co-risk relations emerging. To illustrate this better let us consider ACK (Ackermans \& Van Haaren). The raw data do not show any NCoVaR, however, after linear filtering it poses a very strong threat to the system's recovery (see Table D.3 in D; test statistic of order 6.351 in Scenario 1) and after GARCH filtering it has a weak ex ante effect on the system's risk (test statistic of order 1.329 in Scenario 2). One may speculate that there are some strong purely nonlinear and second moment co-risk effects from ACK on the system variable, which are being partly offset by their linear equivalents. In other words, under normal circumstances ACK does not seem to be an important systemic risk contributor. However, in abnormal times, like a crisis, it reveals its systemic importance.

There is one more finding which we believe is worth pointing out. We confront our results with the official list of Global Systemically Important Banks (G-SIBs), published by the Financial Stability Board (FSB) in 2011. ${ }^{3}$ The FSB recognizes 11 G-SIBs in the euro area. Our sample covers 8 of them, i.e. Banco Bilbao Vizcaya Argentaria (BBV), Banco Santander (SAN), BNP Paribas (BNP), Commerzbank (COM), Deutsche Bank (DEU), Societe Generale (SOC) , UniCredit (Uni) and ING Bank (ING), as a part of the ING Groep. For all of them we confirm their G-SIB status in at least one NCoVaR setting.

\section{Conclusions and discussion}

Conditional Value-at-Risk-Nonlinear Granger Causality, or NCoVaR, is a new methodology of assessing co-risk relations, designed to capture their possible nonlinear Granger causal effects. Our approach distinguishes between two possible scenarios. In the first one, we test what is the role of individual institutions in hampering the recovery of others, given that they are already in distress. In the second scenario, we assess the contribution of individuals to the others' troubles. We derive the regular asymptotic properties of the NCoVaR test for both scenarios and we confirm them numerically.

We apply our methodology to assess the systemic importance of financial institutions in the euro area. Our findings suggest that (i) only a few financial institutions pose a serious ex ante threat to the systemic risk, whereas, given that the system is already in trouble, there are

\footnotetext{
${ }^{3}$ The G-SIBs list is being often updated. In our comparison we consider the most recent version of the list, published on November 11th, 2013.
} 
more institutions which hamper its recovery and (ii) there are intriguing nonlinear structures in its systemic risk profile.

Our study suggests that the most systemically risky institution in our sample is UNI (UniCredit), an Italian bank. In all settings it demonstrates very strong NCoVaR relation to the system. In 2011 it was recognized by the FSB as G-SIB. This analysis confirms its systemical importance, revealing also its nonlinear nature. Interestingly, there are two more companies which demonstrate very strong NCoVaR in 5 out of 6 settings, i.e. ERS (Erste Group Bank), an Austrian bank, and AEG (Aegon), a Dutch insurer. Only the latter was recognized by the FSB to be potentially systemically important, with no official view on the former. However, the former was recognized as systemically important bank for the Austrian financial sector (von Kruechten et al., 2009). Our results point to potential systemic importance of Erste Group Bank in the entire euro area.

NCoVaR might be of great use for macro-prudential policy, however, it has to be tested on other samples and in other periods. It reveals some intriguing phenomena in the co-risk relations. In order to understand them better, a tempting idea is to investigate the underlying nonlinear structures analytically in models of aggregate economy. One may also apply NCoVaR as a mapping tool and bring the risk analysis to the network level. 


\section{References}

V. Acharya. A theory of systemic risk and design of prudential bank regulation. Journal of Financial Stability, 5(3):224-255, 2009.

V. Acharya, L. Pedersen, T. Philippon, and M. Richardson. Measuring systemic risk. Working Paper 1002, New York University, 2010.

T. Adrian and M. K. Brunnermeier. CoVaR. Working Paper 17454, FED New York, 2011.

E. Baeck and W. Brock. A nonparametric test for independence of a multivariate time series. Working Paper 9204, University of Wisconsin - Madison, 1992.

M. Brunnermeier. Deciphering the liquidity and credit crunch 2007-2008. Journal of Economic Perspectives, 23(1):77-100, 2009.

M. Brunnermeier, A. Crocket, C. Goodhart, A. Perssaud, and H. Shin. The Fundamental Principles of Financial Regulation: Geneva Reports on the World Economy. Centre for Economic Policy Research, London, 2009.

J. Caers and M. A. Maes. Identifying tails, bounds and end-points of random variables. Structural Safety, 20(1):1-23, 1998.

M. Chinazzi and G. Fagiolo. Systemic risk, contagion, and financial networks: A survey. LEM Working Paper 2013/08, Sant'Anna School of Advanced Studies, 2013.

M. Denker and G. Keller. On $U$-statistics and v. Mises' statistics for weakly dependent processes. Zeitschrift für Wahrscheinlichkeitstheorie und verwandte Gebiete, 64:505-522, 1983.

C. Diks and V. Panchenko. A note on the Hiemstra-Jones test for Granger non-causality. Studies in Nonlinear Dynamics and Econometrics, 9:1-7, 2005. art. 4.

C. Diks and V. Panchenko. A new statistic and practical guidelines for nonparametric granger causality testing. Journal of Economic Dynamics and Control, 30:1647-1669, 2006.

C. Diks and M. Wolski. Nonlinear granger causality: Guidelines for multivariate analysis. CeNDEF Working Paper 13-15, University of Amsterdam, 2013.

R. Engle. Dynamic conditional correlation: A simple class of multivariate generalized autoregressive conditional heteroskedasticity models. Journal of Business $\&$ Economic Statistics, 20(3):339-350, 2002.

B. B. Francis, M. Mougoua, and V. Panchenko. Is there a symmetric nonlinear causal relationship between large and small firms? Journal of Empirical Finance, 17(1):23-38, 2010.

W. A. Fuller. Introduction to Statistical Time Series (Wiley Series in Probability and Mathematical Statistics). Wiley-Interscience, New York, 1995. 
B. Gao and R.-e. Ren. The topology of a causal network for the Chinese financial system. Physica A: Statistical Mechanics and its Applications, 392(13):2965-2976, 2013.

C. W. J. Granger. Investigating causal relations by econometric models and cross-spectral methods. Econometrica, 37:424-438, 1969.

P. Hall and M. C. Minnotte. High order data sharpening for density estimation. Journal of the Royal Statistical Society. Series B (Statistical Methodology), 64(1):141-157, 2002.

Z. He and A. Krishnamurthy. A macroeconomic framework for quantifying systemic risk. Working Paper 233, National Bank of Belgium, 2012.

C. Hiemstra and J. D. Jones. Testing for linear and nonlinear Granger causality in the stock price - volume relation. Journal of Finance, 49(5):1639-1664, 1994.

X. Huang, H. Zhou, and H. Zhu. Systemic risk contributions. Working Paper 60, Bank for International Settlements, 2010.

K. Jeong, W. K. Härdle, and S. Song. Nonparametric test for causality in quantile. Econometric Theory, 28:861-887, 2012.

M. C. Jones. Estimating densities, quantiles, quantile densities and density quantiles. Annals of the Institute of Statistical Mathematics, 44(4):721-727, 1992.

P. H. Kupiec. Stress testing in a value at risk framework. In M. A. H. Dempster, editor, Risk Management: Value at Risk and Beyond, pages 76-99. Cambridge University Press, Cambridge, 2002.

W. Newey and K. D. West. A simple, positive semi-definite, heteroskedasticity and autocorrelation consistent covariance matrix. Econometrica, 55(3):703-08, 1987.

E. Parzen. On estimation of a probability density function and mode. The Annals of Mathematical Statistics, 33(3):1065-1076, 1962.

P. C. B. Phillips and P. Perron. Testing for a unit root in time series regression. Biometrika, 75(2):335-346, 1988.

R. J. Serfling. Approximation Theorems of Mathematical Statistics. Wiley, New York, 1980.

B. W. Silverman. Density Estimation for Statistics and Data Analysis (Chapman $\mathcal{E}$ Hall/CRC Monographs on Statistics \&3 Applied Probability). Chapman and Hall/CRC, London, 1998.

N. N. Taleb. The Black Swan: The Impact of the Highly Improbable. Random House Trade Paperbacks, New York, 2010.

A. van der Vaart. Asymptotic Statistics. Cambridge University Press, Cambridge, 1998. 
V. von Kruechten, E. Romanova, and M. Schmaus. Austria-based erste group bank ag 'a' rating affirmed on high systemic importance. Discussion Paper 2013/08, Standard \& Poor's Rating Direct, 2009.

M. P. Wand and M. C. Jones. Kernel Smoothing (Chapman \& Hall/CRC Monographs on Statistics \& Applied Probability). Chapman and Hall/CRC, London, 1995.

D. XiaoHua and K. Shiying. International early warning nonlinear model for securities systemic risk based on RBFNN using UDM and BVM. Procedia Engineering, 29(0):1378-1386, 2012. 


\section{A Asymptotic properties of test statistic (Theorem 1)}

We first deal with the properties for the independent sample and consider the dependency later. By symmetrization with respect to two indices, the test statistic in Eq. (10) has a corresponding U-statistic representation of the form

$$
T_{n}\left(\varepsilon_{n}\right) \equiv T_{n}(\varepsilon)=\frac{1}{\left(\begin{array}{c}
n \\
2
\end{array}\right)} \sum_{k=1}^{n} \sum_{p \leq k} \tilde{K}\left(W_{k}, W_{m}\right),
$$

with $W_{k}=\left(Z_{k}, X_{k, l_{i}}^{i}, X_{k, l_{j}}^{j}\right), k=1, \ldots, n$ and kernel given by

$$
\begin{aligned}
\tilde{K}\left(W_{k}, W_{p}\right) & =\frac{\varepsilon^{-d_{Z}-2 d_{X^{i}}-2 d_{X^{j}}}(n-1)}{2 n}\left[K_{k}\left(z_{\gamma}, x_{\gamma}^{i}, x_{*}^{j}\right) K_{p}\left(x_{m}^{i}, x_{*}^{j}\right)\right. \\
& -K_{k}\left(x_{\gamma}^{i}, x_{*}^{j}\right) K_{p}\left(z_{\gamma}, x_{m}^{i}, x_{*}^{j}\right)+K_{p}\left(z_{\gamma}, x_{\gamma}^{i}, x_{*}^{j}\right) K_{k}\left(x_{m}^{i}, x_{*}^{j}\right) \\
& \left.-K_{p}\left(x_{\gamma}^{i}, x_{*}^{j}\right) K_{k}\left(z_{\gamma}, x_{m}^{i}, x_{*}^{j}\right)\right]
\end{aligned}
$$

where for clarity we denote $K_{k}(w)=K\left(\left(w-w_{k}\right) / \varepsilon\right)$ and $d_{Z}, d_{X^{i}}$ and $d_{X^{j}}$ are general representations of the dimensionality of $\mathcal{G}$ and $\mathcal{F}$ operators for particular variables. It is worth to remind here that subscript $n$ in the test statistic refers to its sequence.

The asymptotic properties of the sequence of test statistic can be derived by the projection method (van der Vaart, 1998). From the Háyek's projection lemma we know that the projection of $T_{n}(\varepsilon)-\tau$ on the set of all function of the form $\sum_{k=1}^{n} \kappa_{k}\left(W_{k}\right)$ is given by

$$
\hat{T}_{n}(\varepsilon)=\sum_{k=1}^{n} E\left[\left(T_{n}(\varepsilon)-\tau\right) \mid W_{k}\right]=\frac{2}{n} \sum_{k=1}^{n} \tilde{K}_{1}\left(w_{k}\right),
$$

where

$$
\left.\tilde{K}_{1}\left(w_{k}\right)=E_{W_{p}}\left[\tilde{K}\left(w_{k}, W_{p}\right)\right)\right]-\tau
$$

Projection $\hat{T}_{n}(\varepsilon)$ is mean zero sequence with variance $4 / n \operatorname{Var}\left(\tilde{K}_{1}\left(W_{1}\right)\right)$. By the Central Limit Theorem, one may verify that $\sqrt{n} \hat{T}_{n}(\varepsilon)$ converges in distribution to the normal law with mean 0 and variance given by $4 \operatorname{Var}\left(\tilde{K}_{1}\left(W_{1}\right)\right)$.

Provided that $\operatorname{Var}\left(\hat{T}_{n}(\varepsilon)\right) \rightarrow \operatorname{Var}\left(T_{n}(\varepsilon)\right)$ as $n \rightarrow \infty$, by Slutsky's lemma, we now observe that for a given $\varepsilon$ and given quantiles of any independent finite-variance process $\left(Z_{t}, X_{t, l_{i}}^{i}, X_{t, l_{j}}^{j}\right)$, the sequence $\sqrt{n}\left(T_{n}(\varepsilon)-\tau-\hat{T}_{n}(\varepsilon)\right)$ converges in probability to zero as $n \rightarrow \infty$. What follows, the sequence $\sqrt{n}\left(T_{n}(\varepsilon)-\tau\right)$ converges in distribution to $\mathcal{N}\left(0, \sigma^{2}\right)$, where

$$
\sigma^{2}=4 \zeta_{1}
$$

with $\zeta_{1}=\operatorname{Cov}\left(\tilde{K}\left(W_{1}, W_{2}\right), \tilde{K}\left(W_{1}, W_{2}^{\prime}\right)\right)=\operatorname{Var}\left(\tilde{K}_{1}\left(W_{1}\right)\right)$ 


\section{A.1 Dependence}

Following the reasoning from Denker and Keller (1983), the above asymptotic normality properties of the test statistic, $T_{n}(\varepsilon)$, hold for a weakly dependent process if we take into account the covariance between estimators of particular vectors in the asymptotic variance $\sigma^{2}$,

$$
\sigma^{2}=4\left[\zeta_{1}+2 \sum_{t=2}^{n} \operatorname{Cov}\left(\tilde{K}_{1}\left(W_{1}\right), \tilde{K}_{1}\left(W_{t}\right)\right)\right]
$$

According to the kernel specification, the estimator for $\tilde{K}_{1}\left(W_{k}\right)$ is given by

$$
\hat{K}_{1}\left(W_{k}\right)=\frac{(2 \varepsilon)^{-d_{Z}-2 d_{X^{i}}-2 d_{X^{j}}}}{n} \sum_{p=1}^{n} \tilde{K}\left(W_{k}, W_{p}\right) .
$$

The Newey and West (1987) autocorrelation consistent estimator of $\sigma^{2}$ is then

$$
S_{n}^{2}=\sum_{b=1}^{B} R_{b} \omega_{b}
$$

where $B=\left\lfloor n^{1 / 4}\right\rfloor, R_{b}$ is the sample covariance function of $\hat{K}_{1}\left(W_{b}\right)$ given by

$$
R_{b}=\frac{1}{n-b} \sum_{a=1}^{n-b}\left(\hat{K}_{1}\left(W_{a}\right)-T_{n}(\varepsilon)\right)\left(\hat{K}_{1}\left(W_{a+b}\right)-T_{n}(\varepsilon)\right)
$$

and $\omega_{b}$ is the weight function of the form

$$
\omega_{b}=\left\{\begin{array}{lll}
1, & \text { if } & b=1 \\
2-\frac{2(b-1)}{\tau}, & \text { if } & b>1
\end{array}\right.
$$

For any finite-variance process $\left(Z_{t}, X_{t, l_{i}}^{i}, X_{t, l_{j}}^{j}\right)$, it follows from Denker and Keller (1983) that

$$
\sqrt{n} \frac{\left(T_{n}(\varepsilon)-\tau\right)}{S_{n}} \stackrel{d}{\longrightarrow} \mathcal{N}(0,1)
$$

which completes the proof of Theorem 1.

\section{B Optimal bandwidth sequence (Corollary 1)}

For a given bandwidth $\varepsilon$, the MSE of the test statistic might be rewritten as as sum of variance and squared bias (Wand and Jones, 1995), i.e.

$$
\operatorname{MSE}\left[T_{n}(\varepsilon)\right]=\operatorname{Var}\left(T_{n}(\varepsilon)\right)+\operatorname{Bias}\left(T_{n}(\varepsilon)\right)^{2},
$$


where $\operatorname{Bias}\left(T_{n}(\varepsilon)\right)$ might be calculated explicitly from the Taylor expansion as in Eq. (13) and variance of the test statistic might be represented as $4 S_{n}^{2} / n$ from A.1. Asymptotic covariance terms tend to zero as $n \rightarrow \infty$ so that under the null one might find that the asymptotic variance of $T_{n}(\varepsilon)$ might be decomposed into

$$
\begin{aligned}
\operatorname{Var}\left(T_{n}(\varepsilon)\right) & =\operatorname{Var}\left(\hat{f}_{Z, X^{i}, X^{j}}\left(z_{\gamma}, x_{\gamma}^{i}, x_{*}^{j}\right)\right) \operatorname{Var}\left(\hat{f}_{X^{i}, X^{j}}\left(x_{m}^{i}, x_{*}^{j}\right)\right) \\
& +\operatorname{Var}\left(\hat{f}_{X^{i}, X^{j}}\left(x_{\gamma}^{i}, x_{*}^{j}\right)\right) \operatorname{Var}\left(\hat{f}_{Z, X^{i}, X^{j}}\left(z_{\gamma}, x_{m}^{i}, x_{*}^{j}\right)\right) \\
& +\operatorname{Var}\left(\hat{f}_{X^{i}, X^{j}}\left(x_{m}^{i}, x_{*}^{j}\right)\right) E\left[\hat{f}_{Z, X^{i}, X^{j}}\left(z_{\gamma}, x_{\gamma}^{i}, x_{*}^{j}\right)\right]^{2} \\
& +\operatorname{Var}\left(\hat{f}_{Z, X^{i}, X^{j}}\left(z_{\gamma}, x_{\gamma}^{i}, x_{*}^{j}\right)\right) E\left[\hat{f}_{X^{i}, X^{j}}\left(x_{m}^{i}, x_{*}^{j}\right)\right]^{2} \\
& +\operatorname{Var}\left(\hat{f}_{X^{i}, X^{j}}\left(x_{\gamma}^{i}, x_{*}^{j}\right)\right) E\left[\hat{f}_{Z, X^{i}, X^{j}}\left(z_{\gamma}, x_{m}^{i}, x_{*}^{j}\right)\right]^{2} \\
& +\operatorname{Var}\left(\hat{f}_{Z, X^{i}, X^{j}}\left(z_{\gamma}, x_{m}^{i}, x_{*}^{j}\right)\right) E\left[\hat{f}_{X^{i}, X^{j}}\left(x_{\gamma}^{i}, x_{*}^{j}\right)\right]^{2}+o(1) .
\end{aligned}
$$

One may find that variance and bias of individual density estimators are $o\left(n^{-1} \varepsilon^{-d_{W}}\right)$ and $o\left(\varepsilon^{-2}\right)$, respectively (Silverman, 1998). Therefore, the dominant terms in the asymptotic variance are of order $o\left(n^{-1} \varepsilon^{-d_{Z}-d_{X^{i}}-d_{X^{j}}-4}\right)$.

Taking the first order conditions of the MSE of of individual density estimators, one finds that the optimum rate of convergence of bandwidth parameter is $n^{-1 /\left(d_{W}+4\right)}$. Doing the same for our test statistic, we find that this rate is $n^{-1 /\left(d_{Z}+d_{X^{i}}+d_{X^{j}}\right)}$. Therefore, for any finite dimension, the optimal rate of convergence of the $T_{n}\left(\varepsilon_{n}\right)$ bandwidth is slightly faster than those of individual density estimators but never as fast as $n^{-1}$ which would violate condition (9). Provided that the optimum rate of convergence of the individual estimators is sufficient for the consistency (Silverman, 1998), the optimum rate of $T_{n}\left(\varepsilon_{n}\right)$ guarantees consistency as well.

\section{Data description and results}

\section{Data description and results}

The Euro STOXX Financials Index consists originally of 61 entities. However, only 48 of them cover years 2000-2012 (see Table D.1). For all of them we collect daily equity prices and calculate their log returns accordingly. Data comes from the DataStream and covers period 01/01/2000 till 12/31/2012. All time series are stationary at $1 \%$ significance level, according to both Phillips-Perron and Augmented Dickey-Fuller specifications (Phillips and Perron, 1988; Fuller, 1995). 
Table D.1: List of all entities used in the empirical analysis.

\begin{tabular}{|c|c|c|c|c|}
\hline & Company name/Index & Symbol & Sector & Country \\
\hline 1 & Euro STOXX Financials & STOXX & Aggregate & Aggregate \\
\hline 2 & Ackermans \& Van Haaren & ACK & Financial Services & $\mathrm{BE}$ \\
\hline 3 & Aegon & AEG & Insurance & NL \\
\hline 4 & Ageas & AGE & Insurance & NL \\
\hline 5 & Allianz & ALL & Insurance & $\mathrm{DE}$ \\
\hline 6 & Assicurazioni Generali & ASS & Insurance & IT \\
\hline 7 & $\mathrm{AXA}$ & AXA & Insurance & FR \\
\hline 8 & Bank Of Ireland & BIR & Banks & IR \\
\hline 9 & Bankinter & $\mathrm{BAN}$ & Banks & $\mathrm{ES}$ \\
\hline 10 & Banca Monte Dei Paschi & PAS & Banks & IT \\
\hline 11 & Banca Popolare Di Milano & MIL & Banks & IT \\
\hline 12 & Banca Popolare Di Sondrio & $\mathrm{SON}$ & Banks & IT \\
\hline 13 & Banca Popolare Emilia Romagna & ROM & Banks & IT \\
\hline 14 & BBV Argentaria & $\mathrm{BBV}$ & Banks & ES \\
\hline 15 & Banco Comr. Portugues & POR & Banks & PT \\
\hline 16 & Banco Espirito Santo & ESS & Banks & PT \\
\hline 17 & Banco Popolare & $\mathrm{POP}$ & Banks & IT \\
\hline 18 & Banco Popular Espanol & ESP & Banks & ES \\
\hline 19 & Banco Santander & SAN & Banks & ES \\
\hline 20 & BNP Paribas & $\mathrm{BNP}$ & Banks & FR \\
\hline 21 & CNP Assurances & CNP & Insurance & FR \\
\hline 22 & Cofinimmo & $\mathrm{COF}$ & Real Estate & $\mathrm{BE}$ \\
\hline 23 & Commerzbank & $\mathrm{COM}$ & Banks & $\mathrm{DE}$ \\
\hline 24 & Corio & COR & Real Estate & NL \\
\hline 25 & Deutsche Bank & $\mathrm{DEU}$ & Banks & $\mathrm{DE}$ \\
\hline 26 & Erste Group Bank & ERS & Banks & $\mathrm{AT}$ \\
\hline 27 & Fonciere Des Regions & FON & Real Estate & FR \\
\hline 28 & Gecina & GEC & Real Estate & FR \\
\hline 29 & GBL New & GBL & Financial Services & $\mathrm{BE}$ \\
\hline 30 & Societe Generale & $\mathrm{SOC}$ & Banks & FR \\
\hline 31 & Hannover Ruck. & HAN & Insurance & $\mathrm{DE}$ \\
\hline 32 & ICADE & ICA & Real Estate & FR \\
\hline 33 & Immofinanz & IMM & Real Estate & $\mathrm{AT}$ \\
\hline 34 & ING Groep & ING & Insurance & NL \\
\hline 35 & Intesa Sanpaolo & INT & Banks & $\mathrm{IT}$ \\
\hline 36 & KBC Group & $\mathrm{KBC}$ & Banks & $\mathrm{BE}$ \\
\hline 37 & Klepierre & KLE & Real Estate & FR \\
\hline 38 & Mapfre & MAP & Insurance & ES \\
\hline 39 & Mediobanca & MED & Banks & IT \\
\hline 40 & Muenchener Ruck. & MUE & Insurance & $\mathrm{DE}$ \\
\hline 41 & Natixis & NAT & Banks & FR \\
\hline 42 & Pohjola Pankki & $\mathrm{POH}$ & Banks & FI \\
\hline 43 & Sampo & SAM & Insurance & FI \\
\hline 44 & SCOR & $\mathrm{SCO}$ & Insurance & FR \\
\hline 45 & Unibail-Rodamco & ROD & Real Estate & FR \\
\hline 46 & UniCredit & UNI & Banks & IT \\
\hline 47 & Vienna Insurance Group & VIE & Insurance & AT \\
\hline 48 & Wendel & WEN & Financial Services & FR \\
\hline 49 & Wereldhave & WER & Real Estate & NL \\
\hline
\end{tabular}


Table D.2: NCoVaR from institution $i$ on the system risk in two scenarios in period 01/01/2000 till $12 / 31 / 2012$ for raw returns. Lags determines the optimal number of lags from the VAR specification using the Schwarz-Bayes Information Criterion. Optimal epsilon values calculated from bootstrap. T-val represents the test statistic of NCoVaR from Eq.(10). (*),(**), (***) denotes one-sided p-value statistical significance at $10 \%, 5 \%$ and $1 \%$, respectively.

\begin{tabular}{|c|c|c|c|c|c|c|c|c|}
\hline & \multirow[b]{2}{*}{ Institution $i$} & \multirow[b]{2}{*}{ System variable } & \multicolumn{3}{|c|}{ Scenario 1} & \multicolumn{3}{|c|}{ Scenario 2} \\
\hline & & & Lags & Opt. $\varepsilon$ & T-val & Lags & Opt. $\varepsilon$ & T-val \\
\hline 1 & $\mathrm{ACK}$ & STOXX & 1 & 0.54 & 0.913 & 1 & 0.44 & $1.329 \quad *$ \\
\hline 2 & $\mathrm{AEG}$ & STOXX & 1 & 0.28 & $6.359^{* * *}$ & 1 & 0.4 & $2.855^{* * *}$ \\
\hline 3 & $\mathrm{AGE}$ & STOXX & 4 & 0.6 & 0.213 & 4 & 0.6 & $3.516^{* * *}$ \\
\hline 4 & ALL & STOXX & 1 & 0.38 & 1.45 & 1 & 0.2 & -6.365 \\
\hline 5 & ASS & STOXX & 1 & 0.24 & $6.351^{* * *}$ & 1 & 0.22 & -6.359 \\
\hline 6 & AXA & STOXX & 1 & 0.26 & $6.351^{* * *}$ & 1 & 0.2 & -3.035 \\
\hline 7 & BIR & STOXX & 2 & 0.6 & 0.585 & 2 & 0.6 & $2.611^{* * *}$ \\
\hline 8 & $\mathrm{BAN}$ & STOXX & 1 & 0.38 & -1.262 & 1 & 0.22 & -3.446 \\
\hline 9 & PAS & STOXX & 1 & 0.32 & $6.351^{* * *}$ & 1 & 0.2 & -4.382 \\
\hline 10 & MIL & STOXX & 1 & 0.24 & -6.369 & 1 & 0.2 & -4.477 \\
\hline 11 & $\mathrm{SON}$ & STOXX & 1 & 0.3 & -3.854 & 1 & 0.3 & $4.448 * * *$ \\
\hline 12 & $\mathrm{ROM}$ & STOXX & 1 & 0.48 & 1.571 & 1 & 0.26 & -5.833 \\
\hline 13 & BBV & STOXX & 1 & 0.38 & -1.081 & 1 & 0.2 & -6.361 \\
\hline 14 & POR & STOXX & 1 & 0.26 & -6.351 & 1 & 0.3 & -5.378 \\
\hline 15 & ESS & STOXX & 1 & 0.26 & -6.357 & 1 & 0.2 & -5.44 \\
\hline 16 & POP & STOXX & 1 & 0.32 & -1.551 & 1 & 0.26 & $5.359 * * *$ \\
\hline 17 & ESP & STOXX & 1 & 0.26 & $6.351^{* * *}$ & 1 & 0.2 & -6.364 \\
\hline 18 & SAN & STOXX & 1 & 0.2 & $6.351^{* * *}$ & 1 & 0.42 & 0.673 \\
\hline 19 & BNP & STOXX & 1 & 0.34 & -1.947 & 1 & 0.2 & -3.121 \\
\hline 20 & CNP & STOXX & 1 & 0.3 & 0.78 & 1 & 0.2 & -6.367 \\
\hline 21 & $\mathrm{COF}$ & STOXX & 1 & 0.2 & -6.351 & 1 & 0.2 & -6.365 \\
\hline 22 & COM & STOXX & 1 & 0.26 & $6.351^{* * *}$ & 1 & 0.2 & -6.36 \\
\hline 23 & COR & STOXX & 1 & 0.58 & 0.411 & 1 & 0.22 & -6.375 \\
\hline 24 & DEU & STOXX & 1 & 0.24 & $6.352^{* * *}$ & 1 & 0.24 & -6.371 \\
\hline 25 & ERS & STOXX & 1 & 0.38 & $1.576 \quad *$ & 1 & 0.26 & $3.295 * * *$ \\
\hline 26 & FON & STOXX & 1 & 0.26 & -6.357 & 1 & 0.32 & 0.052 \\
\hline 27 & GEC & STOXX & 1 & 0.24 & -6.362 & 1 & 0.32 & -0.389 \\
\hline 28 & GBL & STOXX & 1 & 0.3 & -6.359 & 1 & 0.42 & 0.088 \\
\hline 29 & SOC & STOXX & 1 & 0.24 & $6.351^{* * *}$ & 1 & 0.22 & -6.364 \\
\hline 30 & HAN & STOXX & 1 & 0.32 & -0.645 & 1 & 0.2 & -3.911 \\
\hline 31 & ICA & STOXX & 1 & 0.24 & -1.474 & 1 & 0.34 & $3.846^{* * *}$ \\
\hline 32 & IMM & STOXX & 2 & 0.6 & -0.316 & 2 & 0.6 & $2.684^{* * *}$ \\
\hline 33 & ING & STOXX & 1 & 0.26 & $6.351^{* * *}$ & 1 & 0.22 & -3.734 \\
\hline 34 & INT & STOXX & 1 & 0.28 & -2.919 & 1 & 0.28 & $4.994 * * *$ \\
\hline 35 & $\mathrm{KBC}$ & STOXX & 1 & 0.26 & $6.352^{* * *}$ & 1 & 0.2 & -6.359 \\
\hline 36 & KLE & STOXX & 1 & 0.24 & $6.351^{* * *}$ & 1 & 0.52 & -0.315 \\
\hline 37 & MAP & STOXX & 1 & 0.26 & -0.444 & 1 & 0.22 & -6.371 \\
\hline 38 & MED & STOXX & 1 & 0.26 & $6.351^{* * *}$ & 1 & 0.2 & -6.361 \\
\hline 39 & MUE & STOXX & 1 & 0.28 & -6.351 & 1 & 0.2 & -6.357 \\
\hline 40 & NAT & STOXX & 1 & 0.26 & $1.417 \quad *$ & 1 & 0.28 & -4.937 \\
\hline 41 & $\mathrm{POH}$ & STOXX & 1 & 0.28 & $6.352^{* * *}$ & 1 & 0.24 & -3.925 \\
\hline 42 & SAM & STOXX & 1 & 0.6 & -0.026 & 1 & 0.28 & -4.666 \\
\hline 43 & SCO & STOXX & 1 & 0.32 & -2.487 & 1 & 0.2 & -6.365 \\
\hline 44 & ROD & STOXX & 1 & 0.52 & 0.239 & 1 & 0.24 & -6.377 \\
\hline 45 & UNI & STOXX & 1 & 0.28 & $6.352^{* * *}$ & 1 & 0.3 & $5.886^{* * *}$ \\
\hline 46 & VIE & STOXX & 1 & 0.32 & $3.747^{* * *}$ & 1 & 0.2 & -6.362 \\
\hline 47 & WEN & STOXX & 1 & 0.24 & $4.733^{* * *}$ & 1 & 0.22 & -6.364 \\
\hline 48 & WER & STOXX & 1 & 0.3 & $3.34^{* * *}$ & 1 & 0.44 & 1.572 \\
\hline
\end{tabular}


Table D.3: NCoVaR from institution $i$ on the system risk in two scenarios in period 01/01/2000 till 12/31/2012 for VAR-filtered returns. Lags determines the optimal number of lags from the VAR specification using the Schwarz-Bayes Information Criterion. Optimal epsilon values calculated from bootstrap. T-val represents the test statistic of NCoVaR from Eq.(10). $\left(^{*}\right),\left(^{* *}\right)$, $(* * *)$ denotes one-sided p-value statistical significance at $10 \%, 5 \%$ and $1 \%$, respectively.

\begin{tabular}{|c|c|c|c|c|c|c|c|c|}
\hline & \multirow[b]{2}{*}{ Institution $i$} & \multirow[b]{2}{*}{ System variable } & \multicolumn{3}{|c|}{ Scenario 1} & \multicolumn{3}{|c|}{ Scenario 2} \\
\hline & & & Lags & Opt. $\varepsilon$ & T-val & Lags & Opt. $\varepsilon$ & T-val \\
\hline 1 & $\mathrm{ACK}$ & STOXX & 1 & 0.26 & $6.351^{* * *}$ & 1 & 0.44 & 1.417 \\
\hline 2 & AEG & STOXX & 1 & 0.24 & $6.355^{* * *}$ & 1 & 0.2 & -6.36 \\
\hline 3 & AGE & STOXX & 1 & 0.38 & 0.983 & 1 & 0.24 & $5.506^{* * *}$ \\
\hline 4 & ALL & STOXX & 1 & 0.36 & 0.247 & 1 & 0.2 & -6.363 \\
\hline 5 & ASS & STOXX & 1 & 0.3 & -2.589 & 1 & 0.22 & -6.361 \\
\hline 6 & AXA & STOXX & 1 & 0.32 & $6.353^{* * *}$ & 1 & 0.2 & -6.358 \\
\hline 7 & BIR & STOXX & 1 & 0.22 & -6.362 & 1 & 0.32 & $4.329 * * *$ \\
\hline 8 & $\mathrm{BAN}$ & STOXX & 1 & 0.28 & -2.625 & 1 & 0.2 & -6.36 \\
\hline 9 & PAS & STOXX & 1 & 0.34 & $6.35^{* * *}$ & 1 & 0.3 & $5.469 * * *$ \\
\hline 10 & MIL & STOXX & 1 & 0.34 & -5.756 & 1 & 0.2 & -3.407 \\
\hline 11 & $\mathrm{SON}$ & STOXX & 1 & 0.36 & $4.458^{* * *}$ & 1 & 0.2 & -5.44 \\
\hline 12 & ROM & STOXX & 1 & 0.34 & $2.491^{* * *}$ & 1 & 0.22 & -5.627 \\
\hline 13 & BBV & STOXX & 1 & 0.2 & $6.35 * * *$ & 1 & 0.2 & -6.36 \\
\hline 14 & POR & STOXX & 1 & 0.32 & -6.36 & 1 & 0.24 & -6.336 \\
\hline 15 & ESS & STOXX & 1 & 0.36 & $1.998 * * *$ & 1 & 0.24 & $5.202^{* * *}$ \\
\hline 16 & POP & STOXX & 1 & 0.34 & -3.085 & 1 & 0.3 & $4.667^{* * *}$ \\
\hline 17 & $\mathrm{ESP}$ & STOXX & 1 & 0.36 & $2.102^{* *}$ & 1 & 0.26 & -6.375 \\
\hline 18 & SAN & STOXX & 1 & 0.2 & $6.351^{* * *}$ & 1 & 0.44 & -0.077 \\
\hline 19 & BNP & STOXX & 1 & 0.22 & $6.35 * * *$ & 1 & 0.2 & -6.356 \\
\hline 20 & CNP & STOXX & 1 & 0.34 & -0.525 & 1 & 0.2 & -6.374 \\
\hline 21 & $\mathrm{COF}$ & STOXX & 1 & 0.34 & -2.344 & 1 & 0.2 & -6.363 \\
\hline 22 & $\mathrm{COM}$ & STOXX & 1 & 0.4 & $3.693 * * *$ & 1 & 0.2 & -6.354 \\
\hline 23 & COR & STOXX & 1 & 0.6 & 0.627 & 1 & 0.24 & -6.38 \\
\hline 24 & DEU & STOXX & 1 & 0.32 & $6.355^{* * *}$ & 1 & 0.22 & -6.366 \\
\hline 25 & ERS & STOXX & 1 & 0.36 & 0.493 & 1 & 0.34 & $1.903^{* *}$ \\
\hline 26 & FON & STOXX & 1 & 0.3 & -6.355 & 1 & 0.3 & 0.633 \\
\hline 27 & GEC & STOXX & 1 & 0.34 & $4.518^{* * *}$ & 1 & 0.46 & -0.53 \\
\hline 28 & GBL & STOXX & 1 & 0.26 & -2.699 & 1 & 0.38 & $3.204^{* * *}$ \\
\hline 29 & $\mathrm{SOC}$ & STOXX & 1 & 0.2 & $6.351^{* * *}$ & 1 & 0.2 & -6.36 \\
\hline 30 & HAN & STOXX & 1 & 0.32 & -6.35 & 1 & 0.2 & -2.984 \\
\hline 31 & ICA & STOXX & 1 & 0.32 & 0.502 & 1 & 0.32 & $4.231^{* * *}$ \\
\hline 32 & IMM & STOXX & 1 & 0.58 & 0.962 & 1 & 0.32 & $4.231^{* * *}$ \\
\hline 33 & ING & STOXX & 1 & 0.26 & $6.35^{* * *}$ & 1 & 0.24 & -6.363 \\
\hline 34 & INT & STOXX & 1 & 0.34 & -4.345 & 1 & 0.28 & $3.23^{* * *}$ \\
\hline 35 & $\mathrm{KBC}$ & STOXX & 1 & 0.24 & $6.351^{* * *}$ & 1 & 0.2 & -6.358 \\
\hline 36 & KLE & STOXX & 1 & 0.36 & $2.593 * * *$ & 1 & 0.36 & $3.947 * * *$ \\
\hline 37 & MAP & STOXX & 1 & 0.48 & $1.519^{*}$ & 1 & 0.2 & -6.364 \\
\hline 38 & MED & STOXX & 1 & 0.2 & $6.35^{* * *}$ & 1 & 0.2 & -6.364 \\
\hline 39 & MUE & STOXX & 1 & 0.24 & -6.35 & 1 & 0.2 & -6.354 \\
\hline 40 & NAT & STOXX & 1 & 0.28 & 1.093 & 1 & 0.3 & -4.97 \\
\hline 41 & $\mathrm{POH}$ & STOXX & 1 & 0.32 & $6.353^{* * *}$ & 1 & 0.44 & 0.314 \\
\hline 42 & SAM & STOXX & 1 & 0.52 & -0.857 & 1 & 0.2 & -6.362 \\
\hline 43 & $\mathrm{SCO}$ & STOXX & 1 & 0.32 & 0.759 & 1 & 0.2 & -6.364 \\
\hline 44 & ROD & STOXX & 1 & 0.5 & -0.401 & 1 & 0.24 & -6.38 \\
\hline 45 & UNI & STOXX & 1 & 0.3 & $6.351^{* * *}$ & 1 & 0.28 & $5.673^{* * *}$ \\
\hline 46 & VIE & STOXX & 1 & 0.34 & 0.611 & 1 & 0.3 & $2.273^{* * *}$ \\
\hline 47 & WEN & STOXX & 1 & 0.26 & $6.368 * * *$ & 1 & 0.2 & -6.359 \\
\hline 48 & WER & STOXX & 1 & 0.32 & -2.424 & 1 & 0.22 & -6.37 \\
\hline
\end{tabular}


Table D.4: NCoVaR from institution $i$ on the system risk in two scenarios in period 01/01/2000 till 12/31/2012 for GARCH-filtered returns. Lags determines the number of lags used in the test. Optimal epsilon values calculated from bootstrap. T-val represents the test statistic of NCoVaR from Eq. $(10) .(*),(* *),(* * *)$ denotes one-sided p-value statistical significance at $10 \%$, $5 \%$ and $1 \%$, respectively.

\begin{tabular}{|c|c|c|c|c|c|c|c|c|}
\hline & \multirow[b]{2}{*}{ Institution $i$} & \multirow[b]{2}{*}{ System variable } & \multicolumn{3}{|c|}{ Scenario 1} & \multicolumn{3}{|c|}{ Scenario 2} \\
\hline & & & Lags & Opt. $\varepsilon$ & T-val & Lags & Opt. $\varepsilon$ & T-val \\
\hline 1 & $\mathrm{ACK}$ & STOXX & 1 & 0.54 & 0.913 & 1 & 0.44 & $1.329^{*}$ \\
\hline 2 & AEG & STOXX & 1 & 0.28 & $6.359 * * *$ & 1 & 0.4 & $2.855^{* * *}$ \\
\hline 3 & AGE & STOXX & 1 & 0.25 & $6.362^{* * *}$ & 1 & 0.23 & -4.646 \\
\hline 4 & ALL & STOXX & 1 & 0.38 & $1.45^{*}$ & 1 & 0.21 & -6.366 \\
\hline 5 & ASS & STOXX & 1 & 0.23 & $6.351^{* * *}$ & 1 & 0.21 & -6.359 \\
\hline 6 & AXA & STOXX & 1 & 0.26 & $6.351^{* * *}$ & 1 & 0.2 & -3.035 \\
\hline 7 & BIR & STOXX & 1 & 0.25 & -4.733 & 1 & 0.21 & $5.319 * * *$ \\
\hline 8 & BAN & STOXX & 1 & 0.38 & -1.262 & 1 & 0.23 & -3.61 \\
\hline 9 & PAS & STOXX & 1 & 0.32 & $6.351^{* * *}$ & 1 & 0.2 & -4.382 \\
\hline 10 & MIL & STOXX & 1 & 0.24 & -6.369 & 1 & 0.21 & -4.477 \\
\hline 11 & $\mathrm{SON}$ & STOXX & 1 & 0.3 & -3.854 & 1 & 0.31 & $4.083^{* * *}$ \\
\hline 12 & ROM & STOXX & 1 & 0.27 & $6.351^{* * *}$ & 1 & 0.27 & -5.883 \\
\hline 13 & BBV & STOXX & 1 & 0.25 & $6.352^{* * *}$ & 1 & 0.21 & -6.362 \\
\hline 14 & POR & STOXX & 1 & 0.26 & -6.351 & 1 & 0.3 & -5.378 \\
\hline 15 & ESS & STOXX & 1 & 0.26 & -6.357 & 1 & 0.21 & -5.771 \\
\hline 16 & POP & STOXX & 1 & 0.29 & -4.344 & 1 & 0.27 & $4.958 * * *$ \\
\hline 17 & ESP & STOXX & 1 & 0.27 & $6.351 * * *$ & 1 & 0.21 & -6.364 \\
\hline 18 & SAN & STOXX & 1 & 0.21 & $6.351^{* * *}$ & 1 & 0.43 & 0.418 \\
\hline 19 & BNP & STOXX & 1 & 0.34 & -1.947 & 1 & 0.2 & -3.121 \\
\hline 20 & CNP & STOXX & 1 & 0.31 & 0.531 & 1 & 0.21 & -6.37 \\
\hline 21 & $\mathrm{COF}$ & STOXX & 1 & 0.2 & -6.351 & 1 & 0.21 & -6.365 \\
\hline 22 & $\mathrm{COM}$ & STOXX & 1 & 0.26 & $6.351^{* * *}$ & 1 & 0.2 & -6.36 \\
\hline 23 & COR & STOXX & 1 & 0.25 & $2.84^{* * *}$ & 1 & 0.21 & -6.371 \\
\hline 24 & DEU & STOXX & 1 & 0.23 & $6.352^{* * *}$ & 1 & 0.23 & -6.37 \\
\hline 25 & ERS & STOXX & 1 & 0.38 & $1.576^{*}$ & 1 & 0.27 & $3.141 * * *$ \\
\hline 26 & FON & STOXX & 1 & 0.26 & -6.357 & 1 & 0.32 & 0.052 \\
\hline 27 & GEC & STOXX & 1 & 0.24 & -6.362 & 1 & 0.33 & -0.46 \\
\hline 28 & GBL & STOXX & 1 & 0.3 & -6.359 & 1 & 0.42 & 0.088 \\
\hline 29 & $\mathrm{SOC}$ & STOXX & 1 & 0.24 & $6.351^{* * *}$ & 1 & 0.22 & -6.364 \\
\hline 30 & HAN & STOXX & 1 & 0.27 & -3.068 & 1 & 0.2 & -3.911 \\
\hline 31 & ICA & STOXX & 1 & 0.25 & -3.335 & 1 & 0.34 & $3.846^{* * *}$ \\
\hline 32 & IMM & STOXX & 1 & 0.22 & -6.361 & 1 & 0.35 & $4.183^{* * *}$ \\
\hline 33 & ING & STOXX & 1 & 0.26 & $6.351^{* * *}$ & 1 & 0.22 & -3.734 \\
\hline 34 & INT & STOXX & 1 & 0.26 & $6.352^{* * *}$ & 1 & 0.2 & -6.359 \\
\hline 35 & $\mathrm{KBC}$ & STOXX & 1 & 0.24 & $6.351^{* * *}$ & 1 & 0.52 & -0.315 \\
\hline 36 & KLE & STOXX & 1 & 0.26 & -0.444 & 1 & 0.22 & -6.371 \\
\hline 37 & MAP & STOXX & 1 & 0.25 & $1.432^{*}$ & 1 & 0.28 & $4.994^{* * *}$ \\
\hline 38 & MED & STOXX & 1 & 0.27 & $6.351^{* * *}$ & 1 & 0.2 & -6.361 \\
\hline 39 & MUE & STOXX & 1 & 0.29 & -6.351 & 1 & 0.2 & -6.357 \\
\hline 40 & NAT & STOXX & 1 & 0.27 & 0.34 & 1 & 0.28 & -4.937 \\
\hline 41 & $\mathrm{POH}$ & STOXX & 1 & 0.29 & $6.352^{* * *}$ & 1 & 0.24 & -3.925 \\
\hline 42 & SAM & STOXX & 1 & 0.59 & -0.147 & 1 & 0.2 & -6.362 \\
\hline 43 & $\mathrm{SCO}$ & STOXX & 1 & 0.32 & -2.487 & 1 & 0.2 & -6.365 \\
\hline 44 & ROD & STOXX & 1 & 0.52 & 0.239 & 1 & 0.23 & -6.375 \\
\hline 45 & UNI & STOXX & 1 & 0.28 & $6.352^{* * *}$ & 1 & 0.3 & $5.886 * * *$ \\
\hline 46 & VIE & STOXX & 1 & 0.32 & $3.747^{* * *}$ & 1 & 0.2 & -6.362 \\
\hline 47 & WEN & STOXX & 1 & 0.25 & $4.869^{* * *}$ & 1 & 0.22 & -6.364 \\
\hline 48 & WER & STOXX & 1 & 0.25 & $6.352^{* * *}$ & 1 & 0.45 & $1.411^{*}$ \\
\hline
\end{tabular}

